\title{
Matrix Completion from a Few Entries
}

\author{
Raghunandan H. Keshavan, Andrea Montanari*†, and Sewoong Oh*
}

September 17, 2009

\begin{abstract}
Let $M$ be an $n \alpha \times n$ matrix of rank $r \ll n$, and assume that a uniformly random subset $E$ of its entries is observed. We describe an efficient algorithm that reconstructs $M$ from $|E|=O(r n)$ observed entries with relative root mean square error

$$
\operatorname{RMSE} \leq C(\alpha)\left(\frac{n r}{|E|}\right)^{1 / 2}
$$

Further, if $r=O(1)$ and $M$ is sufficiently unstructured, then it can be reconstructed exactly from $|E|=O(n \log n)$ entries.

This settles (in the case of bounded rank) a question left open by Candès and Recht and improves over the guarantees for their reconstruction algorithm. The complexity of our algorithm is $O(|E| r \log n)$, which opens the way to its use for massive data sets. In the process of proving these statements, we obtain a generalization of a celebrated result by Friedman-Kahn-Szemerédi and Feige-Ofek on the spectrum of sparse random matrices.
\end{abstract}

\section{Introduction}

Imagine that each of $m$ customers watches and rates a subset of the $n$ movies available through a movie rental service. This yields a dataset of customer-movie pairs $(i, j) \in E \subseteq[m] \times[n]$ and, for each such pair, a rating $M_{i j} \in \mathbb{R}$. The objective of collaborative filtering is to predict the rating for the missing pairs in such a way as to provide targeted suggestions 11 The general question we address here is: Under which conditions do the known ratings provide sufficient information to infer the unknown ones? Can this inference problem be solved efficiently? The second question is particularly important in view of the massive size of actual data sets.

\subsection{Model definition}

A simple mathematical model for such data assumes that the (unknown) matrix of ratings has rank $r \ll m, n$. More precisely, we denote by $M$ the matrix whose entry $(i, j) \in[m] \times[n]$ corresponds to the rating user $i$ would assign to movie $j$. We assume that there exist matrices $U$, of dimensions $m \times r$, and $V$, of dimensions $n \times r$, and a diagonal matrix $\Sigma$, of dimensions $r \times r$ such that

$$
M=U \Sigma V^{T} .
$$

\footnotetext{
${ }^{*}$ Department of Electrical Engineering, Stanford University

${ }^{\dagger}$ Departments of Statistics, Stanford University

${ }^{1}$ Indeed, in 2006, NETFLIX made public such a dataset with $m \approx 5 \cdot 10^{5}, n \approx 2 \cdot 10^{4}$ and $|E| \approx 10^{8}$ and challenged the research community to predict the missing ratings with root mean square error below 0.8563 [Net].
} 
For justification of these assumptions and background on the use of low rank matrices in information retrieval, we refer to BDJ99. Since we are interested in very large data sets, we shall focus on the limit $m, n \rightarrow \infty$ with $m / n=\alpha$ bounded away from 0 and $\infty$.

We further assume that the factors $U, V$ are unstructured. This notion is formalized by the incoherence condition introduced by Candés and Recht [CR08], and defined in Section 2. In particular the incoherence condition is satisfied with high probability if $M=U \Sigma V^{T}$ with $U$ and $V$ uniformly random matrices with $U^{T} U=m \mathbf{1}$ and $V^{T} V=n \mathbf{1}$. Alternatively, incoherence holds if the entries of $U$ and $V$ are i.i.d. bounded random variables.

Out of the $m \times n$ entries of $M$, a subset $E \subseteq[m] \times[n]$ (the user/movie pairs for which a rating is available) is revealed. We let $M^{E}$ be the $m \times n$ matrix that contains the revealed entries of $M$, and is filled with 0's in the other positions

$$
M_{i, j}^{E}=\left\{\begin{aligned}
M_{i, j} & \text { if }(i, j) \in E \\
0 & \text { otherwise }
\end{aligned}\right.
$$

The set $E$ will be uniformly random given its size $|E|$.

\subsection{Algorithm}

A naive algorithm consists of the following projection operation.

Projection. Compute the singular value decomposition (SVD) of $M^{E}$ (with $\sigma_{1} \geq \sigma_{2} \geq \cdots \geq 0$ )

$$
M^{E}=\sum_{i=1}^{\min (m, n)} \sigma_{i} x_{i} y_{i}^{T},
$$

and return the matrix $\mathrm{T}_{r}\left(M^{E}\right)=(m n /|E|) \sum_{i=1}^{r} \sigma_{i} x_{i} y_{i}^{T}$ obtained by setting to 0 all but the $r$ largest singular values. Notice that, apart from the rescaling factor $(m n /|E|), \mathrm{T}_{r}\left(M^{E}\right)$ is the orthogonal projection of $M^{E}$ onto the set of rank-r matrices. The rescaling factor compensates the smaller average size of the entries of $M^{E}$ with respect to $M$.

It turns out that, if $|E|=\Theta(n)$, this algorithm performs very poorly. The reason is that the matrix $M^{E}$ contains columns and rows with $\Theta(\log n / \log \log n)$ non-zero (revealed) entries. The largest singular values of $M^{E}$ are of order $\Theta(\sqrt{\log n / \log \log n})$. The corresponding singular vectors are highly concentrated on high-weight column or row indices (respectively, for left and right singular vectors). Such singular vectors are an artifact of the high-weight columns/rows and do not provide useful information about the hidden entries of $M$. This motivates the definition of the following operation (hereafter the degree of a column or of a row is the number of its revealed entries).

Trimming. Set to zero all columns in $M^{E}$ with degree larger that $2|E| / n$. Set to 0 all rows with degree larger than $2|E| / m$.

Figure 1 shows the singular value distributions of $M^{E}$ and $\widetilde{M}^{E}$ for a random rank-3 matrix $M$. The surprise is that trimming (which amounts to 'throwing out information') makes the underlying rank-3 structure much more apparent. This effect becomes even more important when the number of revealed entries per row/column follows a heavy tail distribution, as for real data.

In terms of the above routines, our algorithm has the following structure.

\begin{tabular}{ll}
\hline SpeCtral Matrix Completion ( matrix $\left.M^{E}\right)$ \\
\hline 1: & Trim $M^{E}$, and let $\widetilde{M}^{E}$ be the output; \\
2: & Project $\widetilde{M}^{E}$ to $\mathrm{T}_{r}\left(\widetilde{M}^{E}\right) ;$ \\
3: & Clean residual errors by minimizing the discrepancy $F(X, Y)$. \\
\hline
\end{tabular}



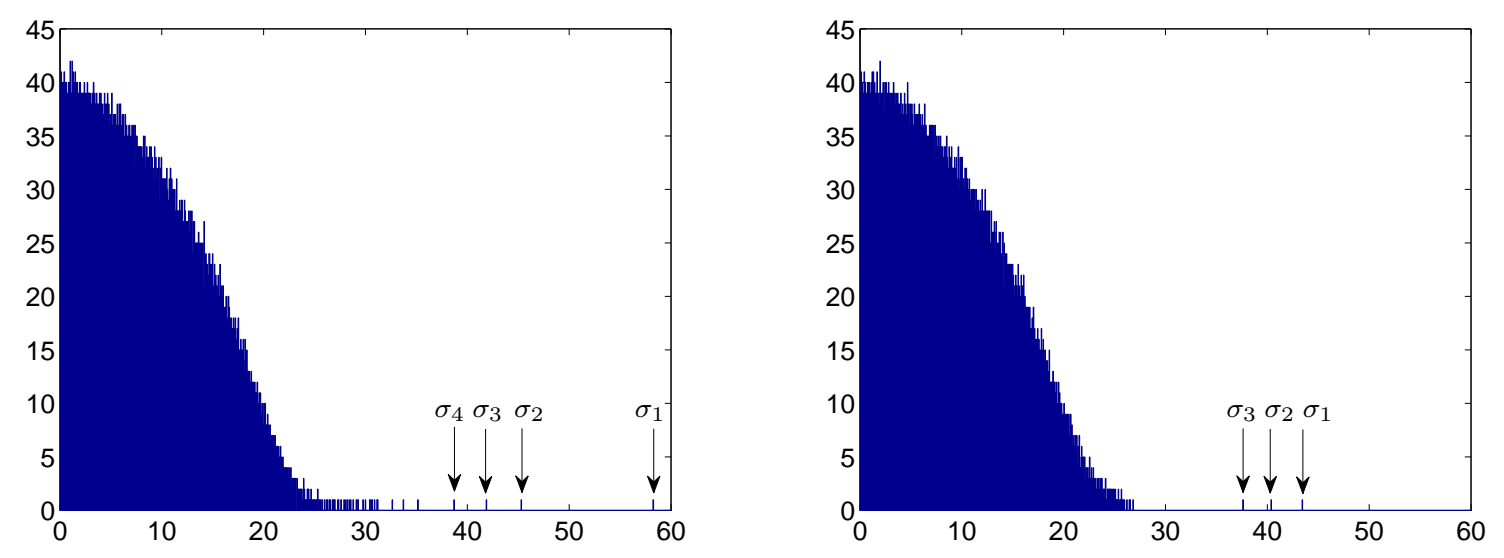

Figure 1: Histogram of the singular values of a partially revealed matrix $M^{E}$ before trimming (left) and after trimming (right) for $10^{4} \times 10^{4}$ random rank-3 matrix $M$ with $\epsilon=30$ and $\Sigma=\operatorname{diag}(1,1.1,1.2)$. After trimming the underlying rank-3 structure becomes clear. Here the number of revealed entries per row follows a heavy tail distribution with $\mathbb{P}\{N=k\}=$ const. $/ k^{3}$.

The last step of the above algorithm allows to reduce (or eliminate) small discrepancies between $\mathrm{T}_{r}\left(\widetilde{M}^{E}\right)$ and $M$, and is described below.

Cleaning. Various implementations are possible, but we found the following one particularly appealing. Given $X \in \mathbb{R}^{m \times r}, Y \in \mathbb{R}^{n \times r}$ with $X^{T} X=m \mathbf{1}$ and $Y^{T} Y=n \mathbf{1}$, we define

$$
\begin{aligned}
F(X, Y) & \equiv \min _{S \in \mathbb{R}^{r \times r}} \mathcal{F}(X, Y, S) \\
\mathcal{F}(X, Y, S) & \equiv \frac{1}{2} \sum_{(i, j) \in E}\left(M_{i j}-\left(X S Y^{T}\right)_{i j}\right)^{2} .
\end{aligned}
$$

The cleaning step consists in writing $\mathrm{T}_{r}\left(\widetilde{M}^{E}\right)=X_{0} S_{0} Y_{0}^{T}$ and minimizing $F(X, Y)$ locally with initial condition $X=X_{0}, Y=Y_{0}$.

Notice that $F(X, Y)$ is easy to evaluate since it is defined by minimizing the quadratic function $S \mapsto \mathcal{F}(X, Y, S)$ over the low-dimensional matrix $S$. Further it depends on $X$ and $Y$ only through their column spaces. In geometric terms, $F$ is a function defined over the cartesian product of two Grassmann manifolds (we refer to Section [6 for background and references). Optimization over Grassmann manifolds is a well understood topic [EAS99] and efficient algorithms (in particular Newton and conjugate gradient) can be applied. To be definite, we assume that gradient descent with line search is used to minimize $F(X, Y)$.

Finally, the implementation proposed here implicitly assumes that the rank $r$ is known. In practice this is a non-issue. Since $r \ll n$, a loop over the value of $r$ can be added at little extra cost. For instance, in collaborative filtering applications, $r$ ranges between 10 and 30.

\subsection{Main results}

Notice that computing $\mathrm{T}_{r}\left(\widetilde{M}^{E}\right)$ only requires to find the first $r$ singular vectors of a sparse matrix. Our main result establishes that this simple procedure achieves arbitrarily small relative root mean 
square error from $O(n r)$ revealed entries. We define the relative root mean square error as

$$
\mathrm{RMSE} \equiv\left[\frac{1}{m n M_{\max }^{2}}\left\|M-\mathrm{T}_{r}\left(\widetilde{M}^{E}\right)\right\|_{\mathrm{F}}^{2}\right]^{1 / 2} .
$$

where we denote by $\|A\|_{F}$ the Frobenius norm of matrix $A$. Notice that the factor $(1 / m n)$ corresponds to the usual normalization by the number of entries and the factor $\left(1 / M_{\max }^{2}\right)$ corresponds to the maximum size of the matrix entries where $M$ satisfies $\left|M_{i, j}\right| \leq M_{\max }$ for all $i$ and $j$.

Theorem 1.1. Assume $M$ to be a rank $r$ matrix of dimension $n \alpha \times n$ that satisfies $\left|M_{i, j}\right| \leq M_{\max }$ for all $i, j$. Then with probability larger than $1-1 / n^{3}$

$$
\frac{1}{m n M_{\max }^{2}}\left\|M-\mathrm{T}_{r}\left(\widetilde{M}^{E}\right)\right\|_{\mathrm{F}}^{2} \leq C \frac{\alpha^{3 / 2} r n}{|E|},
$$

for some numerical constant $C$.

This theorem is proved in Section 3 ,

Notice that the top $r$ singular values and singular vectors of the sparse matrix $\widetilde{M}^{E}$ can be computed efficiently by subspace iteration [Ber92]. Each iteration requires $O(|E| r)$ operations. As proved in Section 3, the $(r+1)$-th singular value is smaller than one half of the $r$-th one. As a consequence, subspace iteration converges exponentially. A simple calculation shows that $O(\log n)$ iterations are sufficient to ensure the error bound mentioned.

The 'cleaning' step in the above pseudocode improves systematically over $\mathrm{T}_{r}\left(\widetilde{M}^{E}\right)$ and, for large enough $|E|$, reconstructs $M$ exactly.

Theorem 1.2. Assume $M$ to be a rank $r$ matrix that satisfies the incoherence conditions $A 1$ and A2 with $\left(\mu_{0}, \mu_{1}\right)$. Let $\mu=\max \left\{\mu_{0}, \mu_{1}\right\}$. Further, assume $\Sigma_{\min } \leq \Sigma_{1}, \ldots, \Sigma_{r} \leq \Sigma_{\max }$ with $\Sigma_{\min }, \Sigma_{\max }$ bounded away from 0 and $\infty$. Then there exists a numerical constant $C^{\prime}$ such that, if

$$
|E| \geq C^{\prime} n r \sqrt{\alpha}\left(\frac{\Sigma_{\max }}{\Sigma_{\min }}\right)^{2} \max \left\{\mu_{0} \log n, \mu^{2} r \sqrt{\alpha}\left(\frac{\Sigma_{\max }}{\Sigma_{\min }}\right)^{4}\right\}
$$

then the cleaning procedure in SPECTRAL MATRIX Completion converges, with high probability, to the matrix $M$.

This theorem is proved in Section 6. The basic intuition is that, for $|E| \geq C^{\prime}(\alpha) n r \max \{\log n, r\}$, $T_{r}\left(\widetilde{M}^{E}\right)$ is so close to $M$ that the cost function is well approximated by a quadratic function.

Theorem 1.1] is optimal: the number of degrees of freedom in $M$ is of order $n r$, without the same number of observations is impossible to fix them. The extra $\log n$ factor in Theorem 1.2 is due to a coupon-collector effect [CR08, KMO08, KOM09]: it is necessary that $E$ contains at least one entry per row and one per column and this happens only for $|E| \geq C n \log n$. As a consequence, for rank $r$ bounded, Theorem 1.2 is optimal. It is suboptimal by a polylogarithmic factor for $r=O(\log n)$.

\subsection{Related work}

Beyond collaborative filtering, low rank models are used for clustering, information retrieval, machine learning, and image processing. In Faz02, the NP-hard problem of finding a matrix of minimum rank satisfying a set of affine constraints was addresses through convex relaxation. This problem is analogous to the problem of finding the sparsest vector satisfying a set of affine constraints, which is at the heart of compressed sensing [Don06, CRT06]. The connection with compressed sensing was 
emphasized in [RFP07, that provided performance guarantees under appropriate conditions on the constraints.

In the case of collaborative filtering, we are interested in finding a matrix $M$ of minimum rank that matches the known entries $\left\{M_{i j}:(i, j) \in E\right\}$. Each known entry thus provides an affine constraint. Candès and Recht [CR08] introduced the incoherent model for $M$. Within this model, they proved that, if $E$ is random, the convex relaxation correctly reconstructs $M$ as long as $|E| \geq$ $C r n^{6 / 5} \log n$. On the other hand, from a purely information theoretic point of view (i.e. disregarding algorithmic considerations), it is clear that $|E|=O(n r)$ observations should allow to reconstruct $M$ with arbitrary precision. Indeed this point was raised in [CR08] and proved in [KMO08], through a counting argument.

The present paper describes an efficient algorithm that reconstructs a rank- $r$ matrix from $O(n r)$ random observations. The most complex component of our algorithm is the SVD in step 2. We were able to treat realistic data sets with $n \approx 10^{5}$. This must be compared with the $O\left(n^{4}\right)$ complexity of semidefinite programming [CR08].

Cai, Candès and Shen CCS08] recently proposed a low-complexity procedure to solve the convex program posed in CR08. Our spectral method is akin to a single step of this procedure, with the important novelty of the trimming step that improves significantly its performances. Our analysis techniques might provide a new tool for characterizing the convex relaxation as well.

Theorem 1.1 can also be compared with a copious line of work in the theoretical computer science literature [FKV04, $\mathrm{AFK}^{+}$01, AM07]. An important motivation in this context is the development of fast algorithms for low-rank approximation. In particular, Achlioptas and McSherry [AM07] prove a theorem analogous to 1.1, but holding only for $|E| \geq(8 \log n)^{4} n$ (in the case of square matrices).

A short account of our results was submitted to the 2009 International Symposium on Information Theory [KOM09]. While the present paper was under completion, Cándes and Tao posted online a preprint proving a theorem analogous to 1.2 [CT09. Once more, their approach is substantially different from ours.

\subsection{Open problems and future directions}

It is worth pointing out some limitations of our results, and interesting research directions:

1. Optimal RMSE with $O(n)$ entries. Numerical simulations with the SPECTRAL Matrix ComPLETION algorithm suggest that the RMSE decays much faster with the number of observations per degree of freedom $(|E| / n r)$, than indicated by Eq. (7)). This improved behavior is a consequence of the cleaning step in the algorithm. It would be important to characterize the decay of RMSE with $(|E| / n r)$.

2. Threshold for exact completion. As pointed out, Theorem 1.2 is order optimal for $r$ bounded. It would nevertheless be useful to derive quantitatively sharp estimates in this regime. A systematic numerical study was initiated in [KMO08]. It appears that available theoretical estimates (including the recent ones in [CT09]) are for larger values of the rank, we expect that our arguments can be strenghtened to prove exact reconstruction for $|E| \geq C^{\prime}(\alpha) n r \log n$ for all values of $r$.

3. More general models. The model studied here and introduced in [CR08] presents obvious limitations. In applications to collaborative filtering, the subset of observed entries $E$ is far from uniformly random. A recent paper [SC09] investigates the uniqueness of the solution of the matrix completion problem for general sets $E$. In applications to fast low-rank approximation, it would be desirable to consider non-incoherent matrices as well (as in [AM07]). 


\section{Incoherence property and some notations}

In order to formalize the notion of incoherence, we write $U=\left[u_{1}, u_{2}, \ldots, u_{r}\right]$ and $V=\left[v_{1}, v_{2}, \ldots, v_{r}\right]$ for the columns of the two factors, with $\left\|u_{i}\right\|=\sqrt{m},\left\|v_{i}\right\|=\sqrt{n}$ and $u_{i}^{T} u_{j}=0, v_{i}^{T} v_{j}=0$ for $i \neq j$ (there is no loss of generality in this, since normalizations can be adsorbed by redefining $\Sigma$ ). We shall further write $\Sigma=\operatorname{diag}\left(\Sigma_{1}, \ldots, \Sigma_{r}\right)$ with $\Sigma_{1} \geq \Sigma_{2} \geq \cdots \geq \Sigma_{r}>0$.

The matrices $U, V$ and $\Sigma$ will be said to be $\left(\mu_{0}, \mu_{1}\right)$-incoherent if they satisfy the following properties:

A1. For all $i \in[m], j \in[n]$, we have $\sum_{k=1}^{r} U_{i, k}^{2} \leq \mu_{0} r, \sum_{k=1}^{r} V_{i, k}^{2} \leq \mu_{0} r$.

A2. For all $i \in[m], j \in[n]$, we have $\left|\sum_{k=1}^{r} U_{i, k}\left(\Sigma_{k} / \Sigma_{1}\right) V_{j, k}\right| \leq \mu_{1} r^{1 / 2}$.

Apart from difference in normalization, these assumptions coincide with the ones in [CR08].

Notice that the second incoherence assumption A2 implies the bounded entry condition in Theorem 1.1 with $M_{\max }=\mu_{1} r^{1 / 2}$. In the following, whenever we write that a property $A$ holds with high probability (w.h.p.), we mean that there exists a function $f(n)=f(n ; \alpha)$ such that $\mathbb{P}(A) \geq 1-f(n)$ and $f(n) \rightarrow 0$. In the case of exact completion (i.e. in the proof of Theorem 1.2) $f(\cdot)$ can also depend on $\mu_{0}, \mu_{1}, \Sigma_{\min }, \Sigma_{\max }$, and $f(n) \rightarrow 0$ for $\mu_{0}, \mu_{1}, \Sigma_{\min }, \Sigma_{\max }$ bounded away from 0 and $\infty$.

Probability is taken with respect to the uniformly random subset $E \subseteq[m] \times[n]$. Define $\epsilon \equiv$ $|E| / \sqrt{m n}$. In the case when $m=n, \epsilon$ corresponds to the average number of revealed entries per row or column. Then, it is convenient to work with a model in which each entry is revealed independently with probability $\epsilon / \sqrt{m n}$. Since, with high probability $|E| \in[\epsilon \sqrt{\alpha} n-A \sqrt{n \log n}, \epsilon \sqrt{\alpha} n+A \sqrt{n \log n}]$, any guarantee on the algorithm performances that holds within one model, holds within the other model as well if we allow for a vanishing shift in $\epsilon$.

Notice that we can assume $m \geq n$, since we can always apply our theorem to the transpose of the matrix $M$. Throughout this paper, therefore, we will assume $\alpha \geq 1$. Finally, we will use $C, C^{\prime}$ etc. to denote numerical constants.

Given a vector $x \in \mathbb{R}^{n},\|x\|$ will denote its Euclidean norm. For a matrix $X \in \mathbb{R}^{n \times n^{\prime}},\|X\|_{F}$ is its Frobenius norm, and $\|X\|_{2}$ its operator norm (i.e. $\left.\|X\|_{2}=\sup _{u \neq 0}\|X u\| /\|u\|\right)$. The standard scalar product between vectors or matrices will sometimes be indicated by $\langle x, y\rangle$ or $\langle X, Y\rangle$, respectively. Finally, we use the standard combinatorics notation $[N]=\{1,2, \ldots, N\}$ to denote the set of first $N$ integers.

\section{Proof of Theorem 1.1 and technical results}

As explained in the previous section, the crucial idea is to consider the singular value decomposition of the trimmed matrix $\widetilde{M}^{E}$ instead of the original matrix $M^{E}$, as in Eq. (3). We shall then redefine $\left\{\sigma_{i}\right\},\left\{x_{i}\right\},\left\{y_{i}\right\}$, by letting

$$
\widetilde{M}^{E}=\sum_{i=1}^{\min (m, n)} \sigma_{i} x_{i} y_{i}^{T} .
$$

Here $\left\|x_{i}\right\|=\left\|y_{i}\right\|=1, x_{i}^{T} x_{j}=y_{i}^{T} y_{j}=0$ for $i \neq j$ and $\sigma_{1} \geq \sigma_{2} \geq \cdots \geq 0$. Our key technical result is that, apart from a trivial rescaling, these singular values are close to the ones of the full matrix $M$.

Lemma 3.1. There exists a numerical constant $C>0$ such that, with probability larger than $1-1 / n^{3}$

$$
\left|\frac{\sigma_{q}}{\epsilon}-\Sigma_{q}\right| \leq C M_{\max } \sqrt{\frac{\alpha}{\epsilon}}
$$


where it is understood that $\Sigma_{q}=0$ for $q>r$.

This result generalizes a celebrated bound on the second eigenvalue of random graphs [FKS89, FO05 and is illustrated in Fig. 1; the spectrum of $\widetilde{M}^{E}$ clearly reveals the rank-3 structure of $M$.

As shown in Section 5, Lemma 3.1 is a direct consequence of the following estimate.

Lemma 3.2. There exists a numerical constant $C>0$ such that, with probability larger than $1-1 / n^{3}$

$$
\left\|\frac{\epsilon}{\sqrt{m n}} M-\widetilde{M}^{E}\right\|_{2} \leq C M_{\max } \sqrt{\alpha \epsilon}
$$

The proof of this lemma is given in Section 4 .

We will now prove Theorem 1.1 .

Proof. (Theorem 1.1) By triangle inequality

$$
\begin{aligned}
\left\|M-\mathrm{T}_{r}\left(\widetilde{M}^{E}\right)\right\|_{2} & \leq\left\|\frac{\sqrt{m n}}{\epsilon} \widetilde{M}^{E}-\mathrm{T}_{r}\left(\widetilde{M}^{E}\right)\right\|_{2}+\left\|M-\frac{\sqrt{m n}}{\epsilon} \widetilde{M}^{E}\right\|_{2} \\
& \leq \sqrt{m n} \sigma_{r+1} / \epsilon+C M_{\max } \sqrt{\alpha m n} / \sqrt{\epsilon} \\
& \leq 2 C M_{\max } \sqrt{\frac{\alpha m n}{\epsilon}}
\end{aligned}
$$

where we used Lemma 3.2 for the second inequality and Lemma 3.1 for the last inequality. Now, for any matrix $A$ of rank at most $2 r,\|A\|_{F} \leq \sqrt{2 r}\|A\|_{2}$, whence

$$
\begin{aligned}
\frac{1}{\sqrt{m n}}\left\|M-\mathrm{T}_{r}\left(\widetilde{M}^{E}\right)\right\|_{F} & \leq \frac{\sqrt{2 r}}{\sqrt{m n}}\left\|M-\mathrm{T}_{r}\left(\widetilde{M}^{E}\right)\right\|_{2} \\
& \leq C^{\prime} M_{\max } \sqrt{\frac{\alpha r}{\epsilon}}
\end{aligned}
$$

The result follows by using $|E|=\epsilon \sqrt{m n}$.

\section{Proof of Lemma 3.2}

We want to show that $\left|x^{T}\left(\widetilde{M}^{E}-\frac{\epsilon}{\sqrt{m n}} M\right) y\right| \leq C M_{\max } \sqrt{\alpha \epsilon}$ for each $x \in \mathbb{R}^{m}, y \in \mathbb{R}^{n}$ such that $\|x\|=\|y\|=1$. Our basic strategy (inspired by [FKS89]) will be the following:

(1) Reduce to $x, y$ belonging to discrete sets $T_{m}, T_{n}$;

(2) Bound the contribution of light couples by applying union bound to these discretized sets, with a large deviation estimate on the random variable $Z$, defined as $Z \equiv \sum_{L} x_{i} \widetilde{M}_{i, j}^{E} y_{j}-\frac{\epsilon}{\sqrt{m n}} x^{T} M y$;

(3) Bound the contribution of heavy couples using bound on the discrepancy of corresponding graph.

The technical challenge is that a worst-case bound on the tail probability of $Z$ is not good enough, and we must keep track of its dependence on $x$ and $y$. The definition of light and heavy couples is provided in the following section.

\subsection{Discretization}

We define

$$
T_{n}=\left\{x \in\left\{\frac{\Delta}{\sqrt{n}} \mathbb{Z}\right\}^{n}:\|x\| \leq 1\right\}
$$


Notice that $T_{n} \subseteq S_{n} \equiv\left\{x \in \mathbb{R}^{n}:\|x\| \leq 1\right\}$. Next remark is proved in [FKS89, FO05], and relates the original problem to the discretized one.

Remark 4.1. Let $R \in \mathbb{R}^{m \times n}$ be a matrix. If $\left|x^{T} R y\right| \leq B$ for all $x \in T_{m}$ and $y \in T_{n}$, then $\left|x^{\prime T} R y^{\prime}\right| \leq(1-\Delta)^{-2} B$ for all $x^{\prime} \in S_{m}$ and $y^{\prime} \in S_{n}$.

Hence it is enough to show that, with high probability, $\left|x^{T}\left(\widetilde{M}^{E}-\frac{\epsilon}{\sqrt{m n}} M\right) y\right| \leq C M_{\max } \sqrt{\alpha \epsilon}$ for all $x \in T_{m}$ and $y \in T_{n}$.

A naive approach would be to apply concentration inequalities directly to the random variable $x^{T}\left(\widetilde{M}^{E}-\frac{\epsilon}{\sqrt{m n}} M\right) y$. This fails because the vectors $x, y$ can contain entries that are much larger than the typical size $O\left(n^{-1 / 2}\right)$. We thus separate two contributions. The first contribution is due to light couples $L \subseteq[m] \times[n]$, defined as

$$
L=\left\{(i, j):\left|x_{i} M_{i j} y_{j}\right| \leq M_{\max }\left(\frac{\epsilon}{m n}\right)^{1 / 2}\right\}
$$

The second contribution is due to its complement $\bar{L}$, which we call heavy couples. We have

$$
\left|x^{T}\left(\widetilde{M}^{E}-\frac{\epsilon}{\sqrt{m n}} M\right) y\right| \leq\left|\sum_{(i, j) \in L} x_{i} \widetilde{M}_{i j}^{E} y_{j}-\frac{\epsilon}{\sqrt{m n}} x^{T} M y\right|+\left|\sum_{(i, j) \in \bar{L}} x_{i} \widetilde{M}_{i j}^{E} y_{j}\right|
$$

In the next two subsections, we will prove that both contributions are upper bounded by $C M_{\max } \sqrt{\alpha \epsilon}$ for all $x \in T_{m}, y \in T_{n}$. Applying Remark 4.1 to $\left|x^{T}\left(\widetilde{M}^{E}-\frac{\epsilon}{\sqrt{m n}} M\right) y\right|$, this proves the thesis.

\subsection{Bounding the contribution of light couples}

Let us define the subset of row and column indices which have not been trimmed as $\mathcal{A}_{l}$ and $\mathcal{A}_{r}$ :

$$
\begin{aligned}
& \mathcal{A}_{l}=\left\{i \in[m]: \operatorname{deg}(i) \leq \frac{2 \epsilon}{\sqrt{\alpha}}\right\} \\
& \mathcal{A}_{r}=\{j \in[n]: \operatorname{deg}(j) \leq 2 \epsilon \sqrt{\alpha}\}
\end{aligned}
$$

where $\operatorname{deg}(\cdot)$ denotes the degree (number of revealed entries) of a row or a column. Notice that $\mathcal{A}=\left(\mathcal{A}_{l}, \mathcal{A}_{r}\right)$ is a function of the random set $E$. It is easy to get a rough estimate of the sizes of $\mathcal{A}_{l}$, $\mathcal{A}_{r}$.

Remark 4.2. There exists $C_{1}$ and $C_{2}$ depending only on $\alpha$ such that, with probability larger than $1-1 / n^{4},\left|\mathcal{A}_{l}\right| \geq m-\max \left\{e^{-C_{1} \epsilon} m, C_{2} \alpha\right\}$, and $\left|\mathcal{A}_{r}\right| \geq n-\max \left\{e^{-C_{1} \epsilon} n, C_{2}\right\}$.

For the proof of this claim, we refer to Appendix $\mathrm{A}$. For any $E \subseteq[m] \times[n]$ and $A=\left(A_{l}, A_{r}\right)$ with $A_{l} \subseteq[m], A_{r} \subseteq[n]$, we define $M^{E, A}$ by setting to zero the entries of $M$ that are not in $E$, those whose row index is not in $A_{l}$, and those whose column index not in $A_{r}$. Consider the event

$$
\mathcal{H}(E, A)=\left\{\exists x, y:\left|\sum_{(i, j) \in L} x_{i} M_{i j}^{E, A} y_{j}-\frac{\epsilon}{\sqrt{m n}} x^{T} M y\right|>C M_{\max } \sqrt{\alpha \epsilon}\right\},
$$


where it is understood that $x$ and $y$ belong, respectively, to $T_{m}$ and $T_{n}$. Note that $\widetilde{M}^{E}=M^{E, \mathcal{A}}$, and hence we want to bound $\mathbb{P}\{\mathcal{H}(E, \mathcal{A})\}$. We proceed as follows

$$
\begin{aligned}
\mathbb{P}\{\mathcal{H}(E, \mathcal{A})\} & =\sum_{A} \mathbb{P}\{\mathcal{H}(E, A), \mathcal{A}=A\} \\
& \leq \sum_{\substack{\left|A_{l}\right| \geq m(1-\delta),\left|A_{r}\right| \geq n(1-\delta)}} \mathbb{P}\{\mathcal{H}(E, A), \mathcal{A}=A\}+\frac{1}{n^{4}} \\
& \leq 2^{(n+m) H(\delta)} \max _{\substack{\left|A_{l}\right| \geq m(1-\delta),\left|A_{r}\right| \geq n(1-\delta)}} \mathbb{P}\{\mathcal{H}(E ; A)\}+\frac{1}{n^{4}}
\end{aligned}
$$

with $\delta \equiv \max \left\{e^{-C_{1} \epsilon}, C_{2} \alpha\right\}$ and $H(x)$ the binary entropy function.

We are now left with the task of bounding $\mathbb{P}\{\mathcal{H}(E ; A)\}$ uniformly over $A$ where $\mathcal{H}$ is defined as in Eq. (13). The key step consists in proving the following tail estimate

Lemma 4.3. Let $x \in S_{m}, y \in S_{n}, Z=\sum_{(i, j) \in L} x_{i} M_{i j}^{E, A} y_{j}-\frac{\epsilon}{\sqrt{m n}} x^{T} M y$, and assume $\left|A_{l}\right| \geq m(1-\delta)$, $\left|A_{r}\right| \geq n(1-\delta)$ with $\delta$ small enough. Then

$$
\mathbb{P}\left(Z>L M_{\max } \sqrt{\epsilon}\right) \leq \exp \left\{-\frac{\sqrt{\alpha}(L-3) n}{2}\right\}
$$

Proof. We begin by bounding the mean of $Z$ as follows (for the proof of this statement we refer to Appendix B]).

Remark 4.4. $|\mathbb{E}[Z]| \leq 2 M_{\max } \sqrt{\epsilon}$.

For $A=\left(A_{l}, A_{r}\right)$, let $M^{A}$ be the matrix obtained from $M$ by setting to zero those entries whose row index is not in $A_{l}$, and those whose column index not in $A_{r}$. Define the potential contribution of the light couples $a_{i j}$ and independent random variables $Z_{i j}$ as

$$
\begin{aligned}
& a_{i j}=\left\{\begin{aligned}
x_{i} M_{i j}^{A} y_{j} & \text { if }\left|x_{i} M_{i j}^{A} y_{j}\right| \leq M_{\max }(\epsilon / m n)^{1 / 2}, \\
0 & \text { otherwise }
\end{aligned}\right. \\
& Z_{i j}=\left\{\begin{aligned}
a_{i, j} & \text { w.p. } \epsilon / \sqrt{m n}, \\
0 & \text { w.p. } 1-\epsilon / \sqrt{m n},
\end{aligned}\right.
\end{aligned}
$$

Let $Z_{1}=\sum_{i, j} Z_{i j}$ so that $Z=Z_{1}-\frac{\epsilon}{\sqrt{m n}} x^{T} M y$. Note that $\sum_{i, j} a_{i j}^{2} \leq \sum_{i, j}\left(x_{i} M_{i j}^{A} y_{j}\right)^{2} \leq M_{\max }^{2}$. Fix $\lambda=\sqrt{m n} / 2 M_{\max } \sqrt{\epsilon}$ so that $\left|\lambda a_{i, j}\right| \leq 1 / 2$, whence $e^{\lambda a_{i j}}-1 \leq \lambda a_{i j}+2\left(\lambda a_{i j}\right)^{2}$. It then follows that

$$
\begin{aligned}
\mathbb{E}\left[e^{\lambda Z}\right] & =\exp \left\{\frac{\epsilon}{\sqrt{m n}}\left(\sum_{i, j} \lambda a_{i, j}+2 \sum_{i, j}\left(\lambda a_{i, j}\right)^{2}\right)-\frac{\lambda \epsilon}{\sqrt{m n}} x^{T} M y\right\} \\
& \leq \exp \left\{\lambda \mathbb{E}[Z]+\frac{\sqrt{m n}}{2}\right\} .
\end{aligned}
$$

The thesis follows by Chernoff bound $\mathbb{P}(Z>a) \leq e^{-\lambda a} \mathbb{E}\left[e^{\lambda Z}\right]$ after simple calculus.

Note that $\mathbb{P}\left(-Z>L M_{\max } \sqrt{\epsilon}\right)$ can also be bounded analogously. We can now finish the upper bound on the light couples contribution. Consider the error event Eq. (13). A simple volume 
calculation shows that $\left|T_{m}\right| \leq(10 / \Delta)^{m}$. We can apply union bound over $T_{m}$ and $T_{n}$ to Eq. (14) to obtain

$$
\begin{aligned}
\mathbb{P}\{\mathcal{H}(E, \mathcal{A})\} & \leq 2 \cdot 2^{(n+m) H(\delta)} \cdot\left(\frac{20}{\Delta}\right)^{n+m} e^{-\frac{(C-3) \sqrt{\alpha} n}{2}}+\frac{1}{n^{4}} \\
& \leq \exp \left\{\log 2+(1+\alpha)(H(\delta) \log 2+\log (20 / \Delta)) n-\frac{(C-3) \sqrt{\alpha} n}{2}\right\}+\frac{1}{n^{4}}
\end{aligned}
$$

Hence, assuming $\alpha \geq 1$, there exists a numerical constant $C^{\prime}$ such that, for $C>C^{\prime} \sqrt{\alpha}$, the first term is of order $e^{-\Theta(n)}$, and this finishes the proof.

\subsection{Bounding the contribution of heavy couples}

Let $Q$ be an $m \times n$ matrix with $Q_{i j}=1$ if $(i, j) \in E$ and $i \notin \mathcal{A}_{r}, j \notin \mathcal{A}_{l}$ (i.e. entry $(i, j)$ is not trimmed by our algorithm), and $Q_{i j}=0$ otherwise. Since $\left|M_{i j}\right| \leq M_{\max }$, the heavy couples satisfy $\left|x_{i} y_{j}\right| \geq \sqrt{\epsilon / m n}$. We then have

$$
\begin{aligned}
\left|\sum_{(i, j) \in \bar{L}} x_{i} \widetilde{M}_{i j}^{E} y_{j}\right| & \leq M_{\max } \sum_{\substack{(i, j) \in \bar{L}\\
}} Q_{i j}\left|x_{i} y_{j}\right| \\
& \leq M_{\max } \sum_{\substack{(i, j) \in E: \\
\left|x_{i} y_{j}\right| \geq \sqrt{\epsilon / m n}}} Q_{i j}\left|x_{i} y_{j}\right|
\end{aligned}
$$

Notice that $Q$ is the adjacency matrix of a random bipartite graph with vertex sets $[m]$ and $[n]$ and maximum degree bounded by $2 \epsilon \max \left(\alpha^{1 / 2}, \alpha^{-1 / 2}\right)$. The following remark strengthens a result of [FO05].

Remark 4.5. Given vectors $x, y$, let $\bar{L}^{\prime}=\left\{(i, j):\left|x_{i} y_{j}\right| \geq C \sqrt{\epsilon / m n}\right\}$. Then there exist a constant $C^{\prime}$ such that, $\sum_{(i, j) \in \bar{L}^{\prime}} Q_{i j}\left|x_{i} y_{j}\right| \leq C^{\prime}\left(\sqrt{\alpha}+\frac{1}{\sqrt{\alpha}}\right) \sqrt{\epsilon}$, for all $x \in T_{m}, y \in T_{n}$ with probability larger than $1-1 / 2 n^{3}$.

For the reader's convenience, a proof of this fact is proposed in Appendix C. The analogous result in [FO05] (for the adjacency matrix of a non-bipartite graph) is proved to hold only with probability larger than $1-e^{-C \epsilon}$. The stronger statement quoted here can be proved using concentration of measure inequalities. The last remark implies that for all $x \in T_{m}, y \in T_{n}$, and $\alpha \geq 1$, the contribution of heavy couples is bounded by $C M_{\max } \sqrt{\alpha \epsilon}$ for some numerical constant $C$ with probability larger than $1-1 / 2 n^{3}$.

\section{Proof of Lemma 3.1}

Recall the variational principle for the singular values.

$$
\begin{aligned}
\sigma_{q} & =\min _{H, \operatorname{dim}(H)=n-q+1} \max _{y \in H,\|y\|=1}\left\|\widetilde{M}^{E} y\right\| \\
& =\max _{H, \operatorname{dim}(H)=q} \min _{y \in H,\|y\|=1}\left\|\widetilde{M}^{E} y\right\| .
\end{aligned}
$$

Here $H$ is understood to be a linear subspace of $\mathbb{R}^{n}$. 
Using Eq. (15) with $H$ the orthogonal complement of $\operatorname{span}\left(v_{1}, \ldots, v_{q-1}\right)$, we have, by Lemma 3.2 ,

$$
\begin{aligned}
\sigma_{q} & \leq \max _{y \in H,\|y\|=1}\left\|\widetilde{M}^{E} y\right\| \\
& \leq \frac{\epsilon}{\sqrt{m n}}\left(\max _{y \in H,\|y\|=1}\|M y\|\right)+\max _{y \in H,\|y\|=\|x\|=1}\left|x^{T}\left(\widetilde{M}^{E}-\frac{\epsilon}{\sqrt{m n}} M\right) y\right| \\
& \leq \epsilon \Sigma_{q}+C M_{\max } \sqrt{\alpha \epsilon}
\end{aligned}
$$

The lower bound is proved analogously, by using Eq. (16) with $H=\operatorname{span}\left(v_{1}, \ldots, v_{q}\right)$.

\section{Minimization on Grassmann manifolds and proof of Theorem 1.2}

The function $F(X, Y)$ defined in Eq. (4) and to be minimized in the last part of the algorithm can naturally be viewed as defined on Grassmann manifolds. Here we recall from [EAS99] a few important facts on the geometry of Grassmann manifold and related optimization algorithms. We then prove Theorem 1.2. Technical calculations are deferred to Sections 7, 8, and to the appendices.

We recall that, for the proof of Theorem 1.2, it is assumed that $\Sigma_{\min }, \Sigma_{\max }$ are bounded away from 0 and $\infty$. Numerical constants are denoted by $C, C^{\prime}$ etc. Finally, throughout this section, we use the notation $X^{(i)} \in \mathbb{R}^{r}$ to refer to the $i$-th row of the matrix $X \in \mathbb{R}^{m \times r}$ or $X \in \mathbb{R}^{n \times r}$.

\subsection{Geometry of the Grassmann manifold}

Denote by $\mathrm{O}(d)$ the orthogonal group of $d \times d$ matrices. The Grassmann manifold is defined as the quotient $\mathrm{G}(n, r) \simeq \mathrm{O}(n) / \mathrm{O}(r) \times \mathrm{O}(n-r)$. In other words, a point in the manifold is the equivalence class of an $n \times r$ orthogonal matrix $A$

$$
[A]=\{A Q: Q \in \mathrm{O}(r)\} .
$$

For consistency with the rest of the paper, we will assume the normalization $A^{T} A=n \mathbf{1}$. To represent a point in $\mathrm{G}(n, r)$, we will use an explicit representative of this form. More abstractly, $\mathrm{G}(n, r)$ is the manifold of $r$-dimensional subspaces of $\mathbb{R}^{n}$.

It is easy to see that $F(X, Y)$ depends on the matrices $X, Y$ only through their equivalence classes $[X],[Y]$. We will therefore interpret it as a function defined on the manifold $\mathrm{M}(m, n) \equiv$ $\mathrm{G}(m, r) \times \mathrm{G}(n, r):$

$$
\begin{aligned}
F: \mathrm{M}(m, n) & \rightarrow \mathbb{R}, \\
([X],[Y]) & \mapsto F(X, Y) .
\end{aligned}
$$

In the following, a point in this manifold will be represented as a pair $\mathbf{x}=(X, Y)$, with $X$ an $n \times r$ orthogonal matrix and $Y$ an $m \times r$ orthogonal matrix. Boldface symbols will be reserved for elements of $\mathrm{M}(m, n)$ or of its tangent space, and we shall use $\mathbf{u}=(U, V)$ for the point corresponding to the matrix $M=U \Sigma V^{T}$ to be reconstructed.

Given $\mathbf{x}=(X, Y) \in \mathrm{M}(m, n)$, the tangent space at $\mathbf{x}$ is denoted by $\mathrm{T}_{\mathbf{x}}$ and can be identified with the vector space of matrix pairs $\mathbf{w}=(W, Z), W \in \mathbb{R}^{m \times r}, Z \in \mathbb{R}^{n \times r}$ such that $W^{T} X=Z^{T} Y=0$. The 'canonical' Riemann metric on the Grassmann manifold corresponds to the usual scalar product $\left\langle W, W^{\prime}\right\rangle \equiv \operatorname{Tr}\left(W^{T} W^{\prime}\right)$. The induced scalar product on $\mathrm{T}_{\mathbf{x}}$ between $\mathbf{w}=(W, Z)$ and $\mathbf{w}^{\prime}=\left(W^{\prime}, Z^{\prime}\right)$ is $\left\langle\mathbf{w}, \mathbf{w}^{\prime}\right\rangle=\left\langle W, W^{\prime}\right\rangle+\left\langle Z, Z^{\prime}\right\rangle$.

This metric induces a canonical notion of distance on $\mathrm{M}(m, n)$ which we denote by $d\left(\mathbf{x}_{1}, \mathbf{x}_{2}\right)$ (geodesic or arc-length distance). If $\mathbf{x}_{1}=\left(X_{1}, Y_{1}\right)$ and $\mathbf{x}_{2}=\left(X_{2}, Y_{2}\right)$ then

$$
d\left(\mathbf{x}_{1}, \mathbf{x}_{2}\right) \equiv \sqrt{d\left(X_{1}, X_{2}\right)^{2}+d\left(Y_{1}, Y_{2}\right)^{2}}
$$


where the arc-length distances $d\left(X_{1}, X_{2}\right), d\left(Y_{1}, Y_{2}\right)$ on the Grassmann manifold can be defined explicitly as follows. Let $\cos \theta=\left(\cos \theta_{1}, \ldots, \cos \theta_{r}\right), \theta_{i} \in[-\pi / 2, \pi / 2]$ be the singular values of $X_{1}^{T} X_{2} / m$. Then

$$
d\left(X_{1}, X_{2}\right)=\|\theta\|_{2} .
$$

The $\theta_{i}$ 's are called the 'principal angles' between the subspaces spanned by the columns of $X_{1}$ and $X_{2}$. It is useful to introduce two equivalent notions of distance:

$$
\begin{array}{ll}
d_{\mathrm{c}}\left(X_{1}, X_{2}\right)=\frac{1}{\sqrt{n}} \min _{Q_{1}, Q_{2} \in \mathrm{O}(r)}\left\|X_{1} Q_{1}-X_{2} Q_{2}\right\|_{F} & \text { (chordal distance), } \\
d_{\mathrm{p}}\left(X_{1}, X_{2}\right)=\frac{1}{\sqrt{2} n}\left\|X_{1} X_{1}^{T}-X_{2} X_{2}^{T}\right\|_{F} & \text { (projection distance). }
\end{array}
$$

Notice that $d_{\mathrm{c}}$ and $d_{\mathrm{p}}$ do not depend on the specific representatives $X_{1}, X_{2}$, but only on the equivalence classes $\left[X_{1}\right]$ and $\left[X_{2}\right]$. Distances on $\mathrm{M}(m, n)$ are defined through Pythagorean theorem, e.g. $d_{\mathrm{c}}\left(\mathbf{x}_{1}, \mathbf{x}_{2}\right)=\sqrt{d_{\mathrm{c}}\left(X_{1}, X_{2}\right)^{2}+d_{\mathrm{c}}\left(Y_{1}, Y_{2}\right)^{2}}$.

Remark 6.1. The geodesic, chordal and projection distance are equivalent, namely

$$
\frac{1}{\pi} d\left(X_{1}, X_{2}\right) \leq \frac{1}{\sqrt{2}} d_{\mathrm{c}}\left(X_{1}, X_{2}\right) \leq d_{\mathrm{p}}\left(X_{1}, X_{2}\right) \leq d_{\mathrm{c}}\left(X_{1}, X_{2}\right) \leq d\left(X_{1}, X_{2}\right)
$$

For the reader's convenience, a proof of this fact is proposed in Appendix D.

An important remark is that geodesics with respect to the canonical Riemann metric admit an explicit and efficiently computable form. Given $\mathbf{u} \in \mathbf{M}(m, n), \mathbf{w} \in \mathbf{T}_{\mathbf{u}}$ the corresponding geodesic is a curve $t \mapsto \mathbf{x}(t)$, with $\mathbf{x}(t)=\mathbf{u}+\mathbf{w} t+O\left(t^{2}\right)$ which minimizes arc-length. If $\mathbf{u}=(U, V)$ and $\mathbf{w}=(W, Z)$ then $\mathbf{x}(t)=(X(t), Y(t))$ where $X(t)$ can be expressed in terms of the singular value decomposition $W=L \Theta R^{T}$ [EAS99]:

$$
X(t)=U R \cos (\Theta t) R^{T}+L \sin (\Theta t) R^{T},
$$

which can be evaluated in time of order $O(n r)$. An analogous expression holds for $Y(t)$.

\subsection{Gradient and incoherence}

The gradient of $F$ at $\mathbf{x}$ is the vector $\operatorname{grad} F(\mathbf{x}) \in \mathrm{T}_{\mathbf{x}}$ such that, for any smooth curve $t \mapsto \mathbf{x}(t) \in$ $\mathrm{M}(m, n)$ with $\mathbf{x}(t)=\mathbf{x}+\mathbf{w} t+O\left(t^{2}\right)$, one has

$$
F(\mathbf{x}(t))=F(\mathbf{x})+\langle\operatorname{grad} F(\mathbf{x}), \mathbf{w}\rangle t+O\left(t^{2}\right) .
$$

In order to write an explicit representation of the gradient of our cost function $F$, it is convenient to introduce the projector operator

$$
\mathcal{P}_{E}(M)_{i j}= \begin{cases}M_{i j} & \text { if }(i, j) \in E \\ 0 & \text { otherwise }\end{cases}
$$

The two components of the gradient are then

$$
\begin{aligned}
& \operatorname{grad} F(\mathbf{x})_{X}=\mathcal{P}_{E}\left(X S Y^{T}-M\right) Y S^{T}-X Q_{X}, \\
& \operatorname{grad} F(\mathbf{x})_{Y}=\mathcal{P}_{E}\left(X S Y^{T}-M\right)^{T} X S-Y Q_{Y},
\end{aligned}
$$

where $Q_{X}, Q_{Y} \in \mathbb{R}^{r \times r}$ are determined by the condition $\operatorname{grad} F(\mathbf{x}) \in \mathrm{T}_{\mathbf{x}}$. This yields

$$
\begin{aligned}
Q_{X} & =\frac{1}{m} X^{T} \mathcal{P}_{E}\left(M-X S Y^{T}\right) Y S^{T} \\
Q_{Y} & =\frac{1}{n} Y^{T} \mathcal{P}_{E}\left(M-X S Y^{T}\right)^{T} X S
\end{aligned}
$$




\subsection{Algorithm}

At this point the gradient descent algorithm is fully specified. It takes as input the factors of $\mathrm{T}_{r}\left(\widetilde{M}^{E}\right)$, to be denoted as $\mathbf{x}_{0}=\left(X_{0}, Y_{0}\right)$, and minimizes a regularized cost function

$$
\begin{aligned}
\widetilde{F}(X, Y) & =F(X, Y)+\rho G(X, Y) \\
& \equiv F(X, Y)+\rho \sum_{i=1}^{m} G_{1}\left(\frac{\left\|X^{(i)}\right\|^{2}}{3 \mu_{0} r}\right)+\rho \sum_{j=1}^{n} G_{1}\left(\frac{\left\|Y^{(j)}\right\|^{2}}{3 \mu_{0} r}\right),
\end{aligned}
$$

where $X^{(i)}$ denotes the $i$-th row of $X$, and $Y^{(j)}$ the $j$-th row of $Y$. The role of the regularization is to force $\mathbf{x}$ to remain incoherent during the execution of the algorithm.

$$
G_{1}(z)= \begin{cases}0 & \text { if } z \leq 1 \\ e^{(z-1)^{2}}-1 & \text { if } z \geq 1\end{cases}
$$

We will take $\rho=n \epsilon$. Notice that $G(X, Y)$ is again naturally defined on the Grassmann manifold, i.e. $G(X, Y)=G\left(X Q, Y Q^{\prime}\right)$ for any $Q, Q^{\prime} \in \mathrm{O}(r)$.

Let

$$
\mathcal{K}\left(\mu^{\prime}\right) \equiv\left\{(X, Y) \text { such that }\left\|X^{(i)}\right\|^{2} \leq \mu^{\prime} r,\left\|Y^{(j)}\right\|^{2} \leq \mu^{\prime} r \text { for all } i \in[m], j \in[n]\right\} .
$$

We have $G(X, Y)=0$ on $\mathcal{K}\left(3 \mu_{0}\right)$. Notice that $\mathbf{u} \in \mathcal{K}\left(\mu_{0}\right)$ by the incoherence property. Also, by the following remark proved in Appendix $\underline{\mathrm{D}}$, we can assume that $\mathbf{x}_{0} \in \mathcal{K}\left(3 \mu_{0}\right)$.

Remark 6.2. Let $U, X \in \mathbb{R}^{n \times r}$ with $U^{T} U=X^{T} X=n \mathbf{1}$ and $U \in \mathcal{K}\left(\mu_{0}\right)$ and $d(X, U) \leq \delta \leq \frac{1}{16}$. Then there exists $X^{\prime \prime} \in \mathbb{R}^{n \times r}$ such that $X^{\prime \prime T} X^{\prime \prime}=n \mathbf{1}, X^{\prime \prime} \in \mathcal{K}\left(3 \mu_{0}\right)$ and $d\left(X^{\prime \prime}, U\right) \leq 4 \delta$. Further, such an $X^{\prime \prime}$ can be computed in a time of $O\left(n r^{2}\right)$.

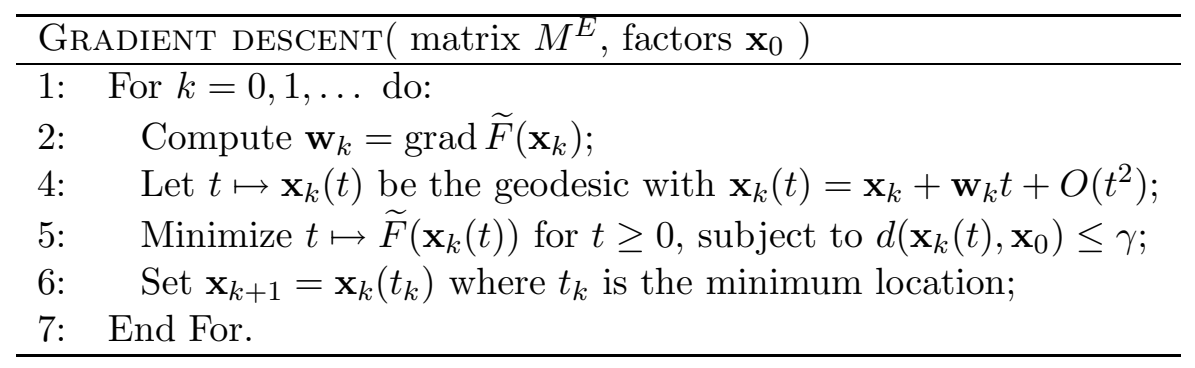

In the above, $\gamma$ must be set in such a way that $d\left(\mathbf{u}, \mathbf{x}_{0}\right) \leq \gamma$. The next remark determines the correct scale.

Remark 6.3. Let $U, X \in \mathbb{R}^{m \times r}$ with $U^{T} U=X^{T} X=m \mathbf{1}, V, Y \in \mathbb{R}^{n \times r}$ with $V^{T} V=Y^{T} Y=n \mathbf{1}$, and $M=U \Sigma V^{T}, \widehat{M}=X S Y^{T}$ for $\Sigma=\operatorname{diag}\left(\Sigma_{1}, \ldots, \Sigma_{r}\right)$ and $S \in \mathbb{R}^{r \times r}$. If $\Sigma_{1}, \ldots, \Sigma_{r} \geq \Sigma_{\min }$, then

$$
d_{\mathrm{p}}(U, X) \leq \frac{1}{\sqrt{2 \alpha} n \Sigma_{\min }}\|M-\widehat{M}\|_{F} \quad, \quad d_{\mathrm{p}}(V, Y) \leq \frac{1}{\sqrt{2 \alpha} n \Sigma_{\min }}\|M-\widehat{M}\|_{F}
$$

As a consequence of this remark and Theorem 1.1, we can assume that $d\left(\mathbf{u}, \mathbf{x}_{0}\right) \leq C\left(\frac{\Sigma_{\max }}{\Sigma_{\min }}\right) \frac{\mu_{1} r \sqrt{\alpha}}{\sqrt{\epsilon}}$. We shall then set $\gamma=C^{\prime}\left(\frac{\Sigma_{\max }}{\Sigma_{\min }}\right) \frac{\mu_{1} r \sqrt{\alpha}}{\sqrt{\epsilon}}$ (the value of $C^{\prime}$ is set in the course of the proof).

Before passing to the proof of Theorem 1.2, it is worth discussing a few important points concerning the gradient descent algorithm. 
(i) The appropriate choice of $\gamma$ might seem to pose a difficulty. In reality, this parameter is introduced only to simplify the proof. We will see that the constraint $d\left(\mathbf{x}_{k}(t), \mathbf{x}_{0}\right) \leq \gamma$ is, with high probability, never saturated.

(ii) Indeed, the line minimization instruction 5 (which might appear complex to implement) can be replaced by a standard step selection procedure, such as the one in [Arm66].

(iii) Similarly, there is no need to know the actual value of $\mu_{0}$ in the regularization term. One can start with $\mu_{0}=1$ and then repeat the optimization doubling it at each step.

(iv) The Hessian of $F$ can be computed explicitly as well. This opens the way to quadratically convergent minimization algorithms (e.g. the Newton method).

\subsection{Proof of Theorem 1.2}

The proof of Theorem 1.2 breaks down in two lemmas. The first one implies that, in a sufficiently small neighborhood of $\mathbf{u}$, the function $\mathbf{x} \mapsto F(\mathbf{x})$ is well approximated by a parabola.

Lemma 6.4. There exists numerical constants $C_{0}, C_{1}, C_{2}$ such that the following happens. Assume $\epsilon \geq C_{0} \mu_{0} \sqrt{\alpha} r \max \left\{\log n ; \mu_{0} r \sqrt{\alpha}\left(\Sigma_{\max } / \Sigma_{\min }\right)^{4}\right\}$ and $\delta \leq \Sigma_{\min } / C_{0} \Sigma_{\max }$. Then

$$
C_{1} \sqrt{\alpha} \Sigma_{\min }^{2} d(\mathbf{x}, \mathbf{u})^{2}+C_{1} \sqrt{\alpha}\|S-\Sigma\|_{F}^{2} \leq \frac{1}{n \epsilon} F(\mathbf{x}) \leq C_{2} \sqrt{\alpha} \Sigma_{\max }^{2} d(\mathbf{x}, \mathbf{u})^{2}
$$

for all $\mathbf{x} \in \mathrm{M}(m, n) \cap \mathcal{K}\left(4 \mu_{0}\right)$ such that $d(\mathbf{x}, \mathbf{u}) \leq \delta$, with probability at least $1-1 / n^{4}$. Here $S \in \mathbb{R}^{r \times r}$ is the matrix realizing the minimum in Eq. (4).

The second Lemma implies that $\mathbf{x} \mapsto F(\mathbf{x})$ does not have any other stationary point (apart from u) within such a neighborhood.

Lemma 6.5. There exists numerical constants $C_{0}, C$ such that the following happens. Assume $\epsilon \geq C_{0} \mu_{0} r \sqrt{\alpha}\left(\Sigma_{\max } / \Sigma_{\min }\right)^{2} \max \left\{\log n ; \mu_{0} r \sqrt{\alpha}\left(\Sigma_{\max } / \Sigma_{\min }\right)^{4}\right\}$ and $\delta \leq \Sigma_{\min } / C_{0} \Sigma_{\max }$. Then

$$
\|\operatorname{grad} \widetilde{F}(\mathbf{x})\|^{2} \geq C n \epsilon^{2} \Sigma_{\min }^{4} d(\mathbf{x}, \mathbf{u})^{2}
$$

for all $\mathbf{x} \in \mathrm{M}(m, n) \cap \mathcal{K}\left(4 \mu_{0}\right)$ such that $d(\mathbf{x}, \mathbf{u}) \leq \delta$, with probability at least $1-1 / n^{4}$.

We can now prove Theorem 1.2 ,

Proof. (Theorem 1.2) Let $\delta>0$ be such that Lemma 6.4 and Lemma 6.5 are verified, and $C_{1}, C_{2}$ be defined as in Lemma 6.4. We further assume $\delta \leq \sqrt{\left(e^{1 / 9}-1\right) / C_{2}}$. Take $\epsilon$ large enough such that, $d\left(\mathbf{u}, \mathbf{x}_{0}\right) \leq \min \left(1,\left(C_{1} / C_{2}\right)^{1 / 2}\left(\Sigma_{\min } / \Sigma_{\max }\right)\right) \delta / 10$. Further, set the algorithm parameter to $\gamma=\delta / 4$.

We make the following claims:

1. $\mathbf{x}_{k} \in \mathcal{K}\left(4 \mu_{0}\right)$ for all $k$.

Indeed $\mathbf{x}_{0} \in \mathcal{K}\left(3 \mu_{0}\right)$ whence $\tilde{F}\left(\mathbf{x}_{0}\right)=F\left(\mathbf{x}_{0}\right) \leq C_{2} \sqrt{\alpha} n \epsilon \Sigma_{\max }^{2} \delta^{2}$. The claim follows because $\widetilde{F}\left(\mathbf{x}_{k}\right)$ is non-increasing and $\widetilde{F}(\mathbf{x}) \geq \rho G(X, Y) \geq n \epsilon \sqrt{\alpha} \Sigma_{\max }^{2}\left(e^{1 / 9}-1\right)$ for $\mathbf{x} \notin \mathcal{K}\left(4 \mu_{0}\right)$, where we choose $\rho$ to be $n \epsilon \sqrt{\alpha} \Sigma_{\max }^{2}$.

2. $d\left(\mathbf{x}_{k}, \mathbf{u}\right) \leq \delta / 10$ for all $k$.

Since we set $\gamma=\delta / 4$, by triangular inequality, we can assume to have $d\left(\mathbf{x}_{k}, \mathbf{u}\right) \leq \delta / 2$. Since $d\left(\mathbf{x}_{0}, \mathbf{u}\right)^{2} \leq\left(C_{1} \Sigma_{\min }^{2} / C_{2} \Sigma_{\max }^{2}\right)(\delta / 10)^{2}$, we have $\widetilde{F}(\mathbf{x}) \geq F(\mathbf{x}) \geq F\left(\mathbf{x}_{0}\right)$ for all $\mathbf{x}$ such that $d(\mathbf{x}, \mathbf{u}) \in[\delta / 10, \delta]$. Since $\widetilde{F}\left(\mathbf{x}_{k}\right)$ is non-increasing and $\widetilde{F}\left(\mathbf{x}_{0}\right)=F\left(\mathbf{x}_{0}\right)$, the claim follows. 
Notice that, by the last observation, the constraint $d\left(\mathbf{x}_{k}(t), \mathbf{x}_{0}\right) \leq \gamma$ is never saturated, and therefore our procedure is just gradient descent with exact line search. Therefore by Arm66 this must converge to the unique stationary point of $\widetilde{F}$ in $\mathcal{K}\left(4 \mu_{0}\right) \cap\{\mathbf{x}: d(\mathbf{x}, \mathbf{u}) \leq \delta / 10\}$, which, by Lemma 6.5, is $\mathbf{u}$.

\section{Proof of Lemma 6.4}

\subsection{A random graph Lemma}

The following Lemma will be used several times in the following.

Lemma 7.1. There exist two numerical constants $C_{1}, C_{2}$ suct that the following happens. If $\epsilon \geq$ $C_{1} \log n$ then, with probability larger than $1-1 / n^{5}$,

$$
\sum_{(i, j) \in E} x_{i} y_{j} \leq \frac{C_{2} \epsilon}{n \sqrt{\alpha}}\|x\|_{1}\|y\|_{1}+C_{2} \sqrt{\alpha \epsilon}\|x\|_{2}\|y\|_{2} .
$$

for all $x \in \mathbb{R}^{m}, y \in \mathbb{R}^{n}$.

Proof. Write $x_{i}=x_{0}+x_{i}^{\prime}$ where $\sum_{i} x_{i}^{\prime}=0$. Then

$$
\sum_{(i, j) \in E} x_{i} y_{j}=x_{0} \sum_{j \in[n]} \operatorname{deg}(j) y_{j}+\sum_{(i, j) \in E} x_{i}^{\prime} y_{j},
$$

where we recall that $\operatorname{deg}(j)=\{i \in[m]:$ such that $(i, j) \in E\}$. Further $\left|x_{0}\right|=\left|\sum_{i} x_{i} / m\right| \leq\|x\|_{1} / m$. The first term is upper bounded by

$$
x_{0} \max _{j \in n} \operatorname{deg}(j)\|y\|_{1} \leq \max _{j \in n} \operatorname{deg}(j)\|x\|_{1}\|y\|_{1} / m .
$$

For $\epsilon \geq C_{1} \log n$, with probability larger than $1-1 / 2 n^{5}$, the maximum degree is bounded by $\left(9 / C_{1}\right) \sqrt{\alpha} \epsilon$ which is of same order as the average degree. Therefore this term is at most $C_{2} \sqrt{\alpha} \epsilon|| x\left\|_{1}\right\| y \|_{1} / m$.

The second term is upper bounded by $C_{2} \sqrt{\alpha \epsilon}\left\|x^{\prime}\right\|_{2}\|y\|_{2}$ using Theorem 1.1 in [FO05] or, equivalently, Theorem 3.1 in the case $r=1$ and $M_{\max }=1$. It can be shown to hold with probability larger than $1-1 / 2 n^{5}$ with a large enough numerical constant $C_{2}$. The thesis follows because $\left\|x^{\prime}\right\|_{2} \leq\|x\|_{2}$.

\subsection{Preliminary facts and estimates}

This subsection contains some remarks that will be useful in the proof of Lemma 6.5 as well.

Let $\mathbf{w}=(W, Z) \in \mathbf{T}_{\mathbf{u}}$, and $t \mapsto(X(t), Y(t))$ be the geodesic such that $(X(t), Y(t))=(U, V)+$ $(W, Z) t+O\left(t^{2}\right)$. By setting $(X, Y)=(X(1), Y(1))$, we establish a one-to-one correspondence between the points $\mathbf{x}$ as in the statement and a neighborhood of the origin in $\mathbf{T}_{\mathbf{u}}$. If we let $W=L \Theta R^{T}$ be the singular value decomposition of $W$ (with $L^{T} L=m \mathbf{1}$ and $R^{T} R=\mathbf{1}$ ), the explicit expression for geodesics in Eq. (25) yields

$$
X=U+\bar{W}, \quad \bar{W}=U R(\cos \Theta-\mathbf{1}) R^{T}+L \sin \Theta R^{T} .
$$

An analogous expression can obviously be written for $Y=V+\bar{Z}$. Notice that, by the equivalence between chordal and canonical distance, Remark 6.1, we have

$$
\frac{1}{m}\|\bar{W}\|_{F}^{2}+\frac{1}{n}\|\bar{Z}\|_{F}^{2} \leq 2 d(\mathbf{u}, \mathbf{x})^{2}
$$


Remark 7.2. If $\mathbf{u} \in \mathcal{K}\left(\mu_{0}\right)$ and $\mathbf{x} \in \mathcal{K}\left(4 \mu_{0}\right)$, then $(\bar{W}, \bar{Z}) \in \mathcal{K}\left(10 \mu_{0}\right)$ and $\mathbf{w}=(W, Z) \in \mathcal{K}\left(5 \pi^{2} \mu_{0} / 2\right)$.

Proof. The first fact follows from $\left\|\bar{W}^{(i)}\right\|^{2} \leq 2\left\|X^{(i)}\right\|^{2}+2\left\|U^{(i)}\right\|^{2}$. In order to prove $\mathbf{w} \in \mathcal{K}\left(5 \pi^{2} \mu_{0} / 2\right)$, we notice that

$$
\begin{aligned}
\left\|W^{(i)}\right\|^{2} & =\left\|\Theta L^{(i)}\right\|^{2} \leq \frac{\pi^{2}}{4}\left\|\sin \Theta L^{(i)}\right\|^{2} \\
& \leq \frac{\pi^{2}}{4}\left\|X^{(i)}-R \cos \Theta R^{T} U^{(i)}\right\|^{2} \leq \frac{\pi^{2}}{2}\left(\left\|X^{(i)}\right\|^{2}+\left\|U^{(i)}\right\|^{2}\right) .
\end{aligned}
$$

The claim follows by showing a similar bound for $\left.\left\|Z^{(i)}\right\|\right|^{2}$.

We next prove a simple a priori estimate.

Remark 7.3. There exist numerical constants $C_{1}, C_{2}$ such that the following holds with probability larger than $1-1 / n^{5}$. If $\epsilon \geq C_{1} \log n$, then for any $(X, Y) \in \mathcal{K}(\mu)$ and $S \in \mathbb{R}^{r \times r}$,

$$
\sum_{(i, j) \in E}\left(X S Y^{T}\right)_{i j}^{2} \leq C_{2}\|S\|_{2}^{2} \sqrt{\alpha} n \epsilon\left(\frac{1}{m}\|X\|_{F}^{2}+\frac{1}{n}\|Y\|_{F}^{2}\right)\left(\frac{1}{m}\|X\|_{F}^{2}+\frac{1}{n}\|Y\|_{F}^{2}+\frac{\mu r \sqrt{\alpha}}{\sqrt{\epsilon}}\right) .
$$

Proof. Using Lemma 7.1, $\sum_{(i, j) \in E}\left(X S Y^{T}\right)_{i j}^{2}$ is upper bounded by

$$
\begin{aligned}
& \sigma_{\max }(S)^{2} \sum_{a, b} \sum_{(i, j) \in E} X_{i a}^{2} Y_{j b}^{2} \\
& \leq \frac{C_{2} \epsilon}{n \sqrt{\alpha}} \sigma_{\max }(S)^{2} \sum_{i, j}\left\|X^{(i)}\right\|^{2}\left\|Y^{(j)}\right\|^{2}+C_{2} \sigma_{\max }(S)^{2} \sqrt{\alpha \epsilon}\left(\sum_{i}\left\|X^{(i)}\right\|^{4}\right)^{1 / 2}\left(\sum_{j}\left\|Y^{(j)}\right\|^{4}\right)^{1 / 2} \\
& \leq \frac{C_{2} \epsilon}{n \sqrt{\alpha}} \sigma_{\max }(S)^{2} \sum_{i, j}\left\|X^{(i)}\right\|^{2}\left\|Y^{(j)}\right\|^{2}+C_{2} \sigma_{\max }(S)^{2} \sqrt{\alpha \epsilon} \mu r\left(\sum_{i}\left\|X^{(i)}\right\|^{2}\right)^{1 / 2}\left(\sum_{j}\left\|Y^{(j)}\right\|^{2}\right)^{1 / 2} \\
& \leq C_{2}\|S\|_{2}^{2} \sqrt{\alpha} n \epsilon\left(\frac{1}{m}\|X\|_{F}^{2}+\frac{1}{n}\|Y\|_{F}^{2}\right)^{2}+C_{2}\|S\|_{2}^{2} \alpha \mu r n \sqrt{\epsilon}\left(\frac{1}{m}\|X\|_{F}^{2}+\frac{1}{n}\|Y\|_{F}^{2}\right),
\end{aligned}
$$

where in the second step we used the incoherence condition. The last step follows from the inequalities $2 a b \leq \alpha(a / \alpha+b)^{2}$ and $2 a b \leq \sqrt{\alpha}\left(a^{2} / \alpha+b^{2}\right)$.

\subsection{The proof}

Proof. (Lemma 6.4) Denote by $S \in \mathbb{R}^{r \times r}$ the matrix realizing the minimum in Eq. (4). We will start by proving a lower bound on $F(\mathbf{x})$ of the form

$$
\frac{1}{n \epsilon} F(\mathbf{x}) \geq C_{1} \sqrt{\alpha} \Sigma_{\min }^{2} d(\mathbf{x}, \mathbf{u})^{2}+C_{1} \sqrt{\alpha}\|S-\Sigma\|_{F}^{2}-C_{1}^{\prime} \sqrt{\alpha} \Sigma_{\max } d(\mathbf{x}, \mathbf{u})^{2}\|S-\Sigma\|_{F},
$$

and an upper bound as in Eq. (37). Together, for $d(\mathbf{x}, \mathbf{u}) \leq \delta \leq 1$, these imply $\|S-\Sigma\|_{F}^{2} \leq$ $C \Sigma_{\max }^{2} d(\mathbf{x}, \mathbf{u})^{2}$, whence the lower bound in Eq. (37) follows for $\delta \leq \Sigma_{\min } / C_{0} \Sigma_{\max }$.

In order to prove the bound (44) we write $X=U+\bar{W}, Y=V+\bar{Z}$, and

$$
\begin{aligned}
F(X, Y) & =\frac{1}{2} \sum_{(i, j) \in E}\left(U(S-\Sigma) V^{T}+U S \bar{Z}^{T}+\bar{W} S V^{T}+\bar{W} S \bar{Z}^{T}\right)_{i j}^{2} \\
& \geq \frac{1}{4} A^{2}-\frac{1}{2} B^{2}
\end{aligned}
$$


where we used the inequality $(1 / 2)(a+b)^{2} \geq\left(a^{2} / 4\right)-\left(b^{2} / 2\right)$, and defined

$$
\begin{aligned}
A^{2} & \equiv \sum_{(i, j) \in E}\left(U(S-\Sigma) V^{T}+U S \bar{Z}^{T}+\bar{W} S V^{T}\right)_{i j}^{2}, \\
B^{2} & \equiv \sum_{(i, j) \in E}\left(\bar{W} S \bar{Z}^{T}\right)_{i j}^{2} .
\end{aligned}
$$

Using Remark 17.3, and Eq. (42) we get

$$
\begin{aligned}
B^{2} & \leq C \sqrt{\alpha} n \epsilon\|S\|_{2}^{2}\left(d(\mathbf{x}, \mathbf{u})^{2}+\frac{\mu_{0} r \sqrt{\alpha}}{\sqrt{\epsilon}}\right) d(\mathbf{x}, \mathbf{u})^{2} \\
& \leq 2 C \sqrt{\alpha} n \epsilon\left(\Sigma_{\max }^{2}+\|S-\Sigma\|_{F}^{2}\right)\left(\delta^{2}+\frac{\mu_{0} r \sqrt{\alpha}}{\sqrt{\epsilon}}\right) d(\mathbf{x}, \mathbf{u})^{2}
\end{aligned}
$$

where the second inequality follows from the inequality $\sigma_{\max }(S)^{2} \leq 2 \Sigma_{\max }^{2}+2\|S-\Sigma\|_{F}^{2}$

By Theorem 4.1 in [CR08], we have $A^{2} \geq(1 / 2) \mathbb{E}\left\{A^{2}\right\}$ with probability larger than $1-1 / n^{5}$ for $\epsilon \geq C \mu_{0} \sqrt{\alpha} r \log n$. Further

$$
\begin{aligned}
\mathbb{E}\left\{A^{2}\right\}= & \frac{\epsilon}{\sqrt{m n}}\left\|U(S-\Sigma) V^{T}+U S \bar{Z}^{T}+\bar{W} S V^{T}\right\|_{F}^{2} \\
= & \frac{\epsilon}{\sqrt{m n}}\left\|U(S-\Sigma) V^{T}\right\|_{F}^{2}+\frac{\epsilon}{\sqrt{m n}}\left\|U S \bar{Z}^{T}\right\|_{F}^{2}+\frac{\epsilon}{\sqrt{m n}}\left\|\bar{W} S V^{T}\right\|_{F}^{2} \\
& +\frac{2 \epsilon}{\sqrt{m n}}\left\langle U S \bar{Z}^{T}, \bar{W} S V^{T}\right\rangle+\frac{2 \epsilon}{\sqrt{m n}}\left\langle U(S-\Sigma) V^{T}, \bar{W} S V^{T}\right\rangle+\frac{2 \epsilon}{\sqrt{m n}}\left\langle U S \bar{Z}^{T}, U(S-\Sigma) V^{T}\right\rangle .
\end{aligned}
$$

Let us call the absolute value of the six terms on the right hand side $E_{1}, \ldots E_{6}$. A simple calculation yields

$$
\begin{aligned}
E_{1} & =n \epsilon \sqrt{\alpha}\|S-\Sigma\|_{F}^{2} \\
E_{2}+E_{3} & \geq n \epsilon \sqrt{\alpha} \sigma_{\min }(S)^{2}\left(\frac{1}{m}\|\bar{W}\|_{F}^{2}+\frac{1}{n}\|\bar{Z}\|_{F}^{2}\right) \geq C^{\prime} \sigma_{\min }(S)^{2} n \epsilon \sqrt{\alpha} d(\mathbf{x}, \mathbf{u})^{2} .
\end{aligned}
$$

The absolute value of the fourth term can be written as

$$
\begin{aligned}
E_{4} & =\frac{2 \epsilon}{n \sqrt{\alpha}}\left|\left\langle U S \bar{Z}^{T}, \bar{W} S V^{T}\right\rangle\right| \leq \frac{2 \epsilon}{n \sqrt{\alpha}} \sigma_{\max }(S)^{2}\left\|\bar{W}^{T} U\right\|_{F}\left\|V^{T} \bar{Z}\right\|_{F} \\
& \leq \frac{2 \epsilon \alpha}{n \sqrt{\alpha}} \sigma_{\max }(S)^{2}\left(\frac{1}{\alpha^{2}}\left\|\bar{W}^{T} U\right\|_{F}^{2}+\left\|V^{T} \bar{Z}\right\|_{F}^{2}\right) .
\end{aligned}
$$

In order proceed, consider Eq. (41). Since by tangency condition $U^{T} L=0$, we have $U^{T} \bar{W}=$ $m R(\cos \Theta-1) R^{T}$ whence

$$
\left\|U^{T} \bar{W}\right\|_{F}=m\|\cos \theta-1\|=\frac{m}{2}\left\|4 \sin ^{2}(\theta / 2)\right\| \leq \frac{m}{2}\|2 \sin (\theta / 2)\|^{2}
$$

(here $\theta=\left(\theta_{1}, \ldots, \theta_{r}\right)$ is the vector containing the diagonal elements of $\Theta$ ). A similar calculation reveals that $\|\bar{W}\|_{F}^{2}=m\|2 \sin (\theta / 2)\|^{2}$ thus proving $\left\|U^{T} \bar{W}\right\|_{F}^{2} \leq\|\bar{W}\|_{F}^{4} / 4 \leq C m \delta^{2}\|\bar{W}\|_{F}^{2}$. The bound $\left\|V^{T} \bar{Z}\right\|_{F}^{2} \leq C n \delta^{2}\|\bar{Z}\|_{F}^{2}$ is proved in the same way, thus yielding

$$
E_{4} \leq C n \epsilon \sqrt{\alpha} \sigma_{\max }(S)^{2} \delta^{2} d(\mathbf{x}, \mathbf{u})^{2} .
$$


By a similar calculation

$$
\begin{aligned}
E_{5} & =\frac{2 \epsilon}{\sqrt{\alpha}} \operatorname{Tr}\left\{(S-\Sigma) S^{T} \bar{W}^{T} U\right\} \leq \frac{2 \epsilon}{\sqrt{\alpha}} \sigma_{\max }\left((S-\Sigma) S^{T}\right)\left\|\bar{W}^{T} U\right\|_{F} \\
& \leq n \epsilon \sqrt{\alpha} \sigma_{\max }(S)\|S-\Sigma\|_{F} d(\mathbf{u}, \mathbf{x})^{2}
\end{aligned}
$$

and analogously

$$
E_{6} \leq n \epsilon \sqrt{\alpha} \sigma_{\max }(S)\|S-\Sigma\|_{F} d(\mathbf{u}, \mathbf{x})^{2}
$$

Combining these estimates, and using $A^{2} \geq \mathbb{E}\left\{A^{2}\right\} / 2$, we get

$$
\begin{aligned}
\frac{1}{n \epsilon} A^{2} \geq \quad C_{1} \sqrt{\alpha}\|S-\Sigma\|_{F}^{2}+C_{1} \sqrt{\alpha} \sigma_{\min }(S)^{2} d(\mathbf{u}, \mathbf{x})^{2} \\
\quad-C_{2} \sqrt{\alpha} \sigma_{\max }(S)^{2} \delta^{2} d(\mathbf{u}, \mathbf{x})^{2}-C_{2} \sqrt{\alpha} \sigma_{\max }(S)\|S-\Sigma\|_{F} d(\mathbf{u}, \mathbf{x})^{2}
\end{aligned}
$$

for some numerical constants $C_{1}, C_{2}>0$. Using the bounds $\sigma_{\min }(S)^{2} \geq \Sigma_{\min }^{2} / 2-\|S-\Sigma\|_{F}^{2}$, $\sigma_{\max }(S)^{2} \leq 2 \Sigma_{\max }^{2}+2\|S-\Sigma\|_{F}^{2}$, and the assumption $d(\mathbf{x}, \mathbf{u}) \leq \delta$ for $\delta \leq \Sigma_{\min } / C_{0} \Sigma_{\max }$, we get the claim (44).

We are now left with the task of proving the upper bound in Eq. (37). We can set $\Sigma=S$, thus obtaining

$$
\begin{aligned}
F(X, Y) & \leq \frac{1}{2} \sum_{(i, j) \in E}\left(U \Sigma \bar{Z}^{T}+\bar{W} \Sigma V^{T}+\bar{W} \Sigma \bar{Z}^{T}\right)_{i j}^{2} \\
& \leq \widehat{A}^{2}+\widehat{B}^{2}
\end{aligned}
$$

where we defined

$$
\begin{aligned}
\widehat{A}^{2} & \equiv \sum_{(i, j) \in E}\left(U \Sigma \bar{Z}^{T}+\bar{W} \Sigma V^{T}\right)_{i j}^{2} \\
\widehat{B}^{2} & \equiv \sum_{(i, j) \in E}\left(\bar{W} \Sigma \bar{Z}^{T}\right)_{i j}^{2} .
\end{aligned}
$$

Bounds for these two quantities are derived as for $A^{2}$ and $B^{2}$. More precisely, by Theorem 4.1 in CR08, we have $\widehat{A}^{2} \leq 2 \mathbb{E}\left\{\widehat{A}^{2}\right\}$ with probability at least $1-1 / n^{5}$ and

$$
\begin{aligned}
\mathbb{E}\left\{\widehat{A}^{2}\right\} & =\frac{\epsilon}{n \sqrt{\alpha}}\left\|\bar{W} \Sigma V^{T}+U \Sigma \bar{Z}^{T}\right\|_{F}^{2} \\
& =\frac{2 \epsilon}{n \sqrt{\alpha}}\left\|\bar{W} \Sigma V^{T}\right\|_{F}^{2}+\frac{2 \epsilon}{n \sqrt{\alpha}}\left\|U \Sigma \bar{Z}^{T}\right\|_{F}^{2} \\
& \leq 2 \sqrt{\alpha} n \epsilon \Sigma_{\max }^{2}\left(\frac{1}{m}\|\bar{W}\|_{F}^{2}+\frac{1}{n}\|\bar{Z}\|_{F}^{2}\right) \leq 4 \sqrt{\alpha} n \epsilon \Sigma_{\max }^{2} d(\mathbf{x}, \mathbf{u})^{2} .
\end{aligned}
$$

$\widehat{B}^{2}$ is bounded similar to $B^{2}$ and we get,

$$
\widehat{B}^{2} \leq C^{\prime} \sqrt{\alpha} n \epsilon \Sigma_{\max }^{2} d(\mathbf{u}, \mathbf{x})^{2} .
$$




\section{Proof of Lemma 6.5}

As in the proof of Lemma 6.4, see Section 7.2, we let $t \mapsto \mathbf{x}(t)=(X(t), Y(t))$ be the geodesic starting at $\mathbf{x}(0)=\mathbf{u}$ with velocity $\dot{\mathbf{x}}(0)=\mathbf{w}=(W, Z) \in \mathrm{T}_{\mathbf{u}}$. We also define $\mathbf{x}=\mathbf{x}(1)=(X, Y)$ with $X=U+\bar{W}$ and $Y=V+\bar{Z}$. Let $\widehat{\mathbf{w}}=\dot{\mathbf{x}}(1)=(\widehat{W}, \widehat{Z})$ be its velocity when passing through $\mathbf{x}$. An explicit expression is obtained in terms of the singular value decomposition of $W$ and $Z$. If we let $W=L \Theta R^{T}$, and differentiate Eq. (25) with respect to $t$ at $t=1$, we obtain

$$
\widehat{W}=-U R \Theta \sin \Theta R^{T}+L \Theta \cos \Theta R^{T} .
$$

An analogous expression holds for $\widehat{Z}$. Since $L^{T} U=0$, we have $\|\widehat{W}\|_{F}^{2}=m\|\Theta \sin \Theta\|_{F}^{2}+m\|\Theta \cos \Theta\|_{F}^{2}=$ $m\|\theta\|^{2}$. Hence 2

$$
\frac{1}{m}\|\widehat{W}\|_{F}^{2}+\frac{1}{n}\|\widehat{Z}\|_{F}^{2}=d(\mathbf{x}, \mathbf{u})^{2}
$$

In order to prove the thesis, it is therefore sufficient to lower bound $\langle\operatorname{grad} \widetilde{F}(\mathbf{x}), \widehat{\mathbf{w}}\rangle$. In the following we will indeed show that

$$
\langle\operatorname{grad} F(\mathbf{x}), \widehat{\mathbf{w}}\rangle \geq C \sqrt{\alpha} n \epsilon \Sigma_{\min }^{2} d(\mathbf{x}, \mathbf{u})^{2},
$$

and $\langle\operatorname{grad} G(\mathbf{x}), \widehat{\mathbf{w}}\rangle \geq 0$, which together imply the thesis by Cauchy-Schwarz inequality.

Let us prove a few preliminary estimates.

Remark 8.1. With the above definitions, $\widehat{\mathbf{w}} \in \mathcal{K}\left((11 / 2) \pi^{2} \mu_{0}\right)$.

Proof. Since $\Theta=\operatorname{diag}\left(\theta_{1}, \ldots, \theta_{r}\right)$ with $\left|\theta_{i}\right| \leq \pi / 2$, we get

$$
\left\|\widehat{W}^{(i)}\right\|^{2} \leq 2\left\|\Theta \sin \Theta R^{T} U^{(i)}\right\|^{2}+2\left\|\Theta \cos \Theta L^{(i)}\right\|^{2} \leq \frac{\pi^{2}}{2}\left\|U^{(i)}\right\|^{2}+2\left\|W^{(i)}\right\|^{2} .
$$

By assumption we have $\left\|U^{(i)}\right\|^{2} \leq \mu_{0} r$ and by Remark 7.2 we have $\left\|W^{(i)}\right\|^{2} \leq 5 \pi^{2} \mu_{0} r / 2$.

One important fact that we will use is that $\widehat{W}$ is well approximated by $W$ or by $\bar{W}$, and $\widehat{Z}$ is well approximated by $Z$ or by $\bar{Z}$. Using Eqs. (41) and (49) we get

$$
\begin{aligned}
\|\widehat{W}\|_{F}^{2} & =\|W\|_{F}^{2}=m\|\theta\|^{2} \\
\|\bar{W}\|_{F}^{2} & =m\|2 \sin \theta / 2\|^{2} \\
\langle\widehat{W}, \bar{W}\rangle & =m \sum_{a=1}^{r} \theta_{a} \sin \theta_{a} \\
\langle\widehat{W}, W\rangle & =m \sum_{a=1}^{r} \theta_{a}^{2} \cos \theta_{a}
\end{aligned}
$$

and therefore

$$
\begin{aligned}
\|\widehat{W}-\bar{W}\|_{F}^{2} & =m \sum_{a=1}^{r}\left[\left(2 \sin \left(\theta_{a} / 2\right)\right)^{2}+\theta_{a}^{2}-2 \theta_{a} \sin \theta_{a}\right] \\
& \leq m \sum_{a=1}^{r}\left(\theta_{a}-2 \sin \left(\theta_{a} / 2\right)\right)^{2} \leq \frac{m}{24^{2}}\|\theta\|^{4} \leq \frac{m}{24^{2}} d(\mathbf{u}, \mathbf{x})^{4} .
\end{aligned}
$$

\footnotetext{
${ }^{2}$ Indeed this conclusion could have been reached immediately, since $t \mapsto \mathbf{x}(t)$ is a geodesic parametrized proportionally to the arclength in th interval $t \in[0,1]$.
} 
Analogously

$$
\|\widehat{W}-W\|_{F}^{2}=n \sum_{a=1}^{r}\left[2 \theta_{a}^{2}-2 \theta_{a}^{2} \cos \theta_{a}\right] \leq m\|\theta\|^{4} \leq m d(\mathbf{u}, \mathbf{x})^{4},
$$

where we used the inequlity $2(1-\cos x) \leq x^{2}$. The last inequality implies in particular

$$
\left\|U^{T} \widehat{W}\right\|_{F}=\left\|U^{T}(W-\widehat{W})\right\|_{F} \leq m d(\mathbf{u}, \mathbf{x})^{2} .
$$

Similar bounds hold of course for $Z, \widehat{Z}, \bar{Z}$ (for instance we have $\left\|V^{T} \widehat{Z}\right\|_{F} \leq n d(\mathbf{u}, \mathbf{x})^{2}$ ). Finally, we shall use repeatedly the fact that $\|S-\Sigma\|_{F}^{2} \leq C \Sigma_{\max }^{2} d(\mathbf{x}, \mathbf{u})^{2}$, which follows from Lemma 6.4. This in turns implies

$$
\begin{aligned}
\sigma_{\max }(S) & \leq \Sigma_{\max }+C \Sigma_{\max } d(\mathbf{x}, \mathbf{u}) \leq 2 \Sigma_{\max } \\
\sigma_{\min }(S) & \geq \Sigma_{\min }-C \Sigma_{\max } d(\mathbf{x}, \mathbf{u}) \geq \frac{1}{2} \Sigma_{\min }
\end{aligned}
$$

where we used the hypothesis $d(\mathbf{x}, \mathbf{u}) \leq \delta=\Sigma_{\min } / C_{0} \Sigma_{\max }$.

\subsection{Lower bound on $\operatorname{grad} F(\mathrm{x})$}

Recalling that $\mathcal{P}_{E}$ is the projector defined in Eq. (27), and using the expression (28), (29), for the gradient, we have

$$
\begin{aligned}
\langle\operatorname{grad} & F(\mathbf{x}), \widehat{\mathbf{w}}\rangle=\left\langle\mathcal{P}_{E}\left(X S Y^{T}-M\right),\left(X S \widehat{Z}^{T}+\widehat{W} S Y^{T}\right)\right\rangle \\
\quad & \left\langle\mathcal{P}_{E}\left(U(S-\Sigma) V^{T}+U S \bar{Z}^{T}+\bar{W} S V^{T}+\bar{W} S \bar{Z}^{T}\right),\left(U S \widehat{Z}^{T}+\widehat{W} S V^{T}+\bar{W} S \widehat{Z}^{T}+\widehat{W} S \bar{Z}^{T}\right)\right\rangle \\
& \geq A-B_{1}-B_{2}-B_{3}
\end{aligned}
$$

where we defined

$$
\begin{aligned}
A & =\left\langle\mathcal{P}_{E}\left(U S \bar{Z}^{T}+\bar{W} S V^{T}\right),\left(U S \widehat{Z}^{T}+\widehat{W} S V^{T}\right)\right\rangle \\
B_{1} & =\left|\left\langle\mathcal{P}_{E}\left(U S \bar{Z}^{T}+\bar{W} S V^{T}\right),\left(\bar{W} S \widehat{Z}^{T}+\widehat{W} S \bar{Z}^{T}\right)\right\rangle\right| \\
B_{2} & =\left|\left\langle\mathcal{P}_{E}\left(U(S-\Sigma) V^{T}+\bar{W} S \bar{Z}^{T}\right),\left(U S \widehat{Z}^{T}+\widehat{W} S V^{T}\right)\right\rangle\right| \\
B_{3} & =\left|\left\langle\mathcal{P}_{E}\left(U(S-\Sigma) V^{T}+\bar{W} S \bar{Z}^{T}\right),\left(\bar{W} S \widehat{Z}^{T}+\widehat{W} S \bar{Z}^{T}\right)\right\rangle\right| .
\end{aligned}
$$

At this point the proof becomes very similar to the one in the previous section and consists in lower bounding $A$ and upper bounding $B_{1}, B_{2}, B_{3}$.

\subsubsection{Lower bound on $A$}

Using Theorem 4.1 in [CR08] we obtain, with probability larger than $1-1 / n^{5}$.

$$
\begin{aligned}
A \geq & \frac{\epsilon}{2 \sqrt{m n}}\left\langle\left(U S \bar{Z}^{T}+\bar{W} S V^{T}\right),\left(U S \widehat{Z}^{T}+\widehat{W} S V^{T}\right)\right\rangle \\
& \geq \frac{1}{2} A_{0}-\frac{1}{2} B_{0}
\end{aligned}
$$

where

$$
\begin{aligned}
& A_{0}=\frac{\epsilon}{2 \sqrt{m n}}\left\|U S \bar{Z}^{T}+\bar{W} S V^{T}\right\|_{F}^{2} \\
& B_{0}=\frac{\epsilon}{2 \sqrt{m n}}\left\|U S \bar{Z}^{T}+\bar{W} S V^{T}\right\|_{F}\left\|U S(\bar{Z}-\widehat{Z})^{T}+(\bar{W}-\widehat{W}) S V^{T}\right\|_{F} .
\end{aligned}
$$


The term $A_{0}$ is lower bounded analogously to $\mathbb{E}\left\{A^{2}\right\}$ in the proof of Lemma 6.4, see Eqs. (46) and (48):

$$
\begin{aligned}
A_{0} & =\frac{\epsilon}{2 \sqrt{m n}}\left\|U S \bar{Z}^{T}+\bar{W} S V^{T}\right\|_{F}^{2} \\
& =\frac{\epsilon}{2 \sqrt{m n}}\left\|U S \bar{Z}^{T}\right\|_{F}^{2}+\frac{\epsilon}{2 \sqrt{m n}}\left\|\bar{W} S V^{T}\right\|_{F}^{2}+\frac{\epsilon}{2 \sqrt{m n}}\left\langle U S \bar{Z}^{T}, \bar{W} S V^{T}\right\rangle \\
& \geq C n \epsilon\left(\sqrt{\alpha} \sigma_{\min }(S)^{2}-\sqrt{\alpha} \delta^{2} \sigma_{\max }(S)^{2}\right) d(\mathbf{x}, \mathbf{u})^{2} \geq C \sqrt{\alpha} n \epsilon \Sigma_{\min }^{2} d(\mathbf{x}, \mathbf{u})^{2}
\end{aligned}
$$

where we used the bounds (60), (61) and the hypothesis $d(\mathbf{x}, \mathbf{u}) \leq \delta=\Sigma_{\min } / C_{0} \Sigma_{\max }$.

As for the second term we notice that

$$
\begin{aligned}
\frac{B_{0}^{2}}{A_{0}} & \leq n \epsilon \sqrt{\alpha}\left(\frac{1}{m}\|S(\bar{W}-\widehat{W})\|_{F}^{2}+\frac{1}{n}\left\|S^{T}(\bar{Z}-\widehat{Z})\right\|_{F}^{2}\right) \\
& \leq n \epsilon \sqrt{\alpha} \sigma_{\max }(S)^{2}\left(\frac{1}{m}\|\bar{W}-\widehat{W}\|_{F}^{2}+\frac{1}{n}\|\bar{Z}-\widehat{Z}\|_{F}^{2}\right) \leq C n \epsilon \sqrt{\alpha} \Sigma_{\max }^{2} d(\mathbf{x}, \mathbf{u})^{4}
\end{aligned}
$$

where, in the last step, we used the estimate (57) and the analogous one for $\|\bar{Z}-\widehat{Z}\|_{F}^{2}$. Therefore for $d(\mathbf{x}, \mathbf{u}) \leq \delta \leq \Sigma_{\min } / C_{0} \Sigma_{\max }$ and $C_{0}$ large enough $A_{0}>2 B_{0}$, whence

$$
A \geq C \sqrt{\alpha} n \epsilon \Sigma_{\min }^{2} d(\mathbf{x}, \mathbf{u})^{2} .
$$

\subsubsection{Upper bound on $B_{1}$}

We begin by noting that $B_{1}$ can be bounded above by the sum of four terms of the form $B_{1}^{\prime}=$ $\left|\left\langle\mathcal{P}_{E}\left(U S \bar{Z}^{T}\right), \bar{W} S \widehat{Z}^{T}\right\rangle\right|$. We show that $B_{1}^{\prime}<A / 100$. The other terms are bounded similarly.

Using Remark 7.3, we have

$$
\begin{aligned}
\left\|\mathcal{P}_{E}\left(\bar{W} S \widehat{Z}^{T}\right)\right\|_{F}^{2} \leq & C \frac{\epsilon}{\sqrt{m n}}\|\bar{W}\|_{F}^{2}\left\|S \widehat{Z}^{T}\right\|_{F}^{2}+C^{\prime} \sqrt{\epsilon} \mu_{0} r \sqrt{\alpha} \Sigma_{\max }\|\bar{W}\|_{F}\|S \widehat{Z}\|_{F} \\
\leq & 2 C \frac{\epsilon}{\sqrt{m n}}\|\bar{W}\|_{F}^{2}\left\|S \bar{Z}^{T}\right\|_{F}^{2}+2 C \frac{\epsilon}{\sqrt{m n}}\|\bar{W}\|_{F}^{2}\left\|S(\widehat{Z}-\bar{Z})^{T}\right\|_{F}^{2} \\
& +C^{\prime} \sqrt{\epsilon} \mu_{0} r \sqrt{\alpha} \Sigma_{\max }\|\bar{W}\|_{F}\|S \bar{Z}\|_{F}+C^{\prime} \sqrt{\epsilon} \mu_{0} r \sqrt{\alpha} \Sigma_{\max }\|\bar{W}\|_{F}\|S(\widehat{Z}-\bar{Z})\|_{F} \\
\leq & C^{\prime \prime} A\left(\delta^{2}+\frac{\mu_{0} r \sqrt{\alpha} \Sigma_{\max }}{\sqrt{\epsilon} \Sigma_{\min }}\right)
\end{aligned}
$$

where we have used $\frac{\epsilon m}{\sqrt{m n}}\left\|S \bar{Z}^{T}\right\|_{F}^{2} \leq 3 A_{0} \leq 12 A$ from Section 8.1.1. Therefore we have,

$$
\begin{aligned}
B_{1}^{\prime 2} & \leq\left\|\mathcal{P}_{E}\left(U S \bar{Z}^{T}\right)\right\|_{F}^{2}\left\|\mathcal{P}_{E}\left(\bar{W} S \widehat{Z}^{T}\right)\right\|_{F}^{2} \\
& \leq C \frac{\epsilon}{\sqrt{m n}}\left\|U S \bar{Z}^{T}\right\|_{F}^{2} A\left(\delta^{2}+\frac{\mu_{0} r \sqrt{\alpha} \Sigma_{\max }}{\sqrt{\epsilon} \Sigma_{\min }}\right) \\
& \leq C^{\prime} A^{2}\left(\delta^{2}+\frac{\mu_{0} r \sqrt{\alpha} \Sigma_{\max }}{\sqrt{\epsilon} \Sigma_{\min }}\right)
\end{aligned}
$$

The thesis follows for $\delta$ and $\epsilon$ as in the hypothesis. 


\subsubsection{Upper bound on $B_{2}$}

We have

$$
\begin{aligned}
B_{2} \leq & \left|\left\langle\mathcal{P}_{E}\left(U S \widehat{Z}^{T}+\widehat{W} S V^{T}\right), \bar{W} S \bar{Z}^{T}\right\rangle\right|+\left|\left\langle\mathcal{P}_{E}\left(U S \widehat{Z}^{T}\right), U(S-\Sigma) V^{T}\right\rangle\right| \\
& \quad+\left|\left\langle\mathcal{P}_{E}\left(\widehat{W} S V^{T}\right), U(S-\Sigma) V^{T}\right\rangle\right| \\
\equiv & B_{2}^{\prime}+B_{2}^{\prime \prime}+B_{2}^{\prime \prime \prime} .
\end{aligned}
$$

We claim that each of these three terms is smaller than $A / 30$, whence $B_{2} \leq A / 10$.

The upper bound on $B_{2}^{\prime}$ is obtained similarly to the one on $B_{1}$ to get $B_{2}^{\prime} \leq A / 30$.

Consider now $B_{2}^{\prime \prime}$. By Theorem 4.1 in [CR08,

$$
B_{2}^{\prime \prime} \leq \frac{\epsilon}{\sqrt{m n}}\left|\left\langle U(S-\Sigma) V^{T}, U S \widehat{Z}^{T}\right\rangle\right|+C \frac{\epsilon}{\sqrt{m n}} \sqrt{\frac{\mu_{0} n r \alpha \log n}{n \sqrt{\alpha} \epsilon}}|| U(S-\Sigma) V^{T}\left\|_{F}\right\| U S \widehat{Z} \|_{F}
$$

To bound the second term, observe

$$
\begin{aligned}
\left\|U S \widehat{Z}^{T}\right\|_{F} & \leq\left\|U S \bar{Z}^{T}\right\|_{F}+\left\|U S(\widehat{Z}-\bar{Z})^{T}\right\|_{F} \\
& \leq\left\|U S \bar{Z}^{T}\right\|_{F}+\Sigma_{\max } \sqrt{m n} d(\mathbf{x}, \mathbf{u})^{2}
\end{aligned}
$$

Also, $\frac{\epsilon}{\sqrt{m n}}\left\|U S \bar{Z}^{T}\right\|_{F}^{2} \leq 3 A_{0} \leq 12 A$ from Section 8.1.1. Combining these, we have that the second term in $B_{2}^{\prime \prime}$ is smaller than $A / 60$ for $\epsilon$ as in the hypothesis.

To bound the first term in $B_{2}^{\prime \prime}$,

$$
\begin{aligned}
\left|\left\langle U(S-\Sigma) V^{T}, U S \widehat{Z}^{T}\right\rangle\right| & =\left|\left\langle U(S-\Sigma)(Y-V)^{T}, U S \widehat{Z}^{T}\right\rangle\right| \\
& \leq\left\|U(S-\Sigma) \bar{Z}^{T}\right\|_{F}\left\|U S \bar{Z}^{T}\right\|_{F}+\left\|U(S-\Sigma) \bar{Z}^{T}\right\|_{F} \| U S\left(\bar{Z}-\widehat{Z} \|_{F}\right.
\end{aligned}
$$

Therefore

$$
\begin{aligned}
B_{2}^{\prime \prime} & \leq \frac{\epsilon}{\sqrt{m n}}\|U(S-\Sigma) \bar{Z}\|_{F}\|U S \bar{Z}\|_{F}+\epsilon n \sqrt{\alpha} \Sigma_{\max }^{2} d(\mathbf{x}, \mathbf{u})^{4}+A / 60 \\
& \leq \frac{\epsilon}{\sqrt{m n}}\|U(S-\Sigma) \bar{Z}\|_{F}\|U S \bar{Z}\|_{F}+A / 40
\end{aligned}
$$

for $d(\mathbf{x}, \mathbf{u}) \leq \delta$ as in the hypothesis.

We are now left with upper bounding $\widetilde{B}_{2}^{\prime \prime} \equiv \frac{\epsilon}{\sqrt{m n}}\|U(S-\Sigma) \bar{Z}\|_{F}\|U S \bar{Z}\|_{F}$.

$$
\begin{aligned}
\widetilde{B}_{2}^{\prime \prime 2} & \leq\left(\frac{\epsilon}{\sqrt{m n}}\left\|U(S-\Sigma) \bar{Z}^{T}\right\|_{F}^{2}\right)\left(\frac{\epsilon}{\sqrt{m n}}\left\|U S \bar{Z}^{T}\right\|_{F}^{2}\right) \\
& \leq\left(\epsilon n \sqrt{\alpha} \Sigma_{\max }^{2} d(\mathbf{x}, \mathbf{u})^{4}\right)\left(\frac{\epsilon}{\sqrt{m n}}\left\|U S \bar{Z}^{T}\right\|_{F}^{2}\right)
\end{aligned}
$$

Also from the lower bound on A, we have, $\frac{\epsilon}{\sqrt{m n}}\left\|U S \bar{Z}^{T}\right\|_{F}^{2} \leq 3 A_{0} \leq 12 A$. Using $d(\mathbf{x}, \mathbf{u}) \leq \delta$, we have $\widetilde{B}_{2}^{\prime \prime} \leq A / 120$ for $\delta$ as in the hypothesis. This proves the desired result. The bound on $B_{2}^{\prime \prime \prime}$ is calculated analogously. 


\subsubsection{Upper bound on $B_{3}$}

Finally for the last term it is sufficient to use a crude bound

$$
B_{3} \leq 4\left(\left\|\mathcal{P}_{E}\left(\bar{W} S \widehat{Z}^{T}\right)\right\|_{F}+\left\|\mathcal{P}_{E}\left(\widehat{W} S \bar{Z}^{T}\right)\right\|_{F}\right)\left(\left\|\mathcal{P}_{E}\left(U(S-\Sigma) V^{T}\right)\right\|_{F}+\left\|\mathcal{P}_{E}\left(\bar{W} S \bar{Z}^{T}\right)\right\|_{F}\right),
$$

The terms of the form $\left\|\mathcal{P}_{E}\left(\bar{W} S \widehat{Z}^{T}\right)\right\|_{F}$ are all estimated as in Section 8.1.2, Also, by Theorem 4.1 of CR08

$$
\begin{aligned}
\left\|\mathcal{P}_{E}\left(U(S-\Sigma) V^{T}\right)\right\|_{F} & \leq C \frac{\epsilon}{\sqrt{m n}}\left\|U(S-\Sigma) V^{T}\right\|_{F}^{2} \\
& \leq C n \epsilon \sqrt{\alpha} \Sigma_{\max }^{2} d(\mathbf{x}, \mathbf{u})^{2}
\end{aligned}
$$

Combining these estimates with the $\delta$ and the $\epsilon$ in the hypothesis, we get $B_{3} \leq A / 10$

\subsection{Lower bound on $\operatorname{grad} G(\mathrm{x})$}

By the definition of $G$ in Eq. (33), we have

$$
\langle\operatorname{grad} G(\mathbf{x}), \widehat{\mathbf{w}}\rangle=\frac{1}{\mu_{0} r} \sum_{i=1}^{m} G_{1}^{\prime}\left(\frac{\left\|X^{(i)}\right\|^{2}}{3 \mu_{0} r}\right)\left\langle X^{(i)}, \widehat{W}^{(i)}\right\rangle+\frac{1}{\mu_{0} r} \sum_{j=1}^{n} G_{1}^{\prime}\left(\frac{\left\|Y^{(i)}\right\|^{2}}{3 \mu_{0} r}\right)\left\langle Y^{(i)}, \widehat{Z}^{(i)}\right\rangle .
$$

It is therefore sufficient to show that if $\left\|X^{(i)}\right\|^{2}>3 \mu_{0} r$, then $\left\langle X^{(i)}, \widehat{W}^{(i)}\right\rangle>0$, and if $\left\|Y^{(j)}\right\|^{2}>$ $3 \mu_{0} r$, then $\left\langle Y^{(j)}, \widehat{Z}^{(j)}\right\rangle>0$. We will just consider the first statement, the second being completely symmetrical.

From the explicit expressions (41) and (49) we get

$$
\begin{aligned}
X^{(i)} & =R\left\{\cos \Theta R^{T} U^{(i)}+\sin \Theta L^{(i)}\right\}, \\
\widehat{W}^{(i)} & =R\left\{\Theta \cos \Theta L^{(i)}-\Theta \sin \Theta R^{T} U^{(i)}\right\} .
\end{aligned}
$$

From the first expression it follows that

$$
\left\|\sin \Theta L^{(i)}\right\|^{2} \leq\left\|X^{(i)}\right\|^{2}+\left\|\cos \Theta R^{T} U^{(i)}\right\|^{2} \leq 5 \mu_{0} r .
$$

On the other hand, by taking the difference of Eqs. (67) and (68) we have

$$
\begin{aligned}
\left\|X^{(i)}-\widehat{W}^{(i)}\right\| & \leq\left\|(\sin \Theta-\Theta \cos \Theta) L^{(i)}\right\|+\left\|(\cos \Theta+\Theta \sin \Theta) R^{T} U^{(i)}\right\| \\
& \leq \max _{i}\left(\theta_{i}^{2}\right)\left\|\sin \Theta L^{(i)}\right\|+\frac{\pi}{2}\left\|U^{(i)}\right\| \leq \delta \sqrt{4 \mu_{0} r}+\frac{\pi}{2} \sqrt{\mu_{0} r} .
\end{aligned}
$$

where we used the inequality $(\sin \omega-\omega \cos \omega) \leq \omega^{2} \sin \omega$ valid for $\omega \in[0, \pi / 2]$. For $\delta$ small enough we have therefore $\left\|X^{(i)}-\widehat{W}^{(i)}\right\| \leq(99 / 100) \sqrt{3 \mu_{0} r}$. To conclude, for $\left\|X^{(i)}\right\| \geq 3 \mu_{0} r$

$$
\left\langle X^{(i)}, \widehat{W}^{(i)}\right\rangle \geq\left\|X^{(i)}\right\|^{2}-\left\|X^{(i)}\right\|\left\|X^{(i)}-\widehat{W}^{(i)}\right\| \geq\left\|X^{(i)}\right\|\left(\sqrt{3 \mu_{0} r}-(99 / 100) \sqrt{3 \mu_{0} r}\right) \geq 0 .
$$

\section{Acknowledgements}

We thank Emmanuel Candés and Benjamin Recht for stimulating discussions on the subject of this paper. This work was partially supported by a Terman fellowship and an NSF CAREER award (CCF-0743978). 


\section{A Proof of Remark 4.2}

The proof is a generalization of analogous result in [FO05], which is proved to hold only with probability larger than $1-e^{-C \epsilon}$. The stronger statement quoted here can be proved using concentration of measure inequalities.

First, we apply Chernoff bound to the event $\left\{\left|\overline{\mathcal{A}}_{l}\right|>\max \left\{e^{-C_{1} \epsilon} m, C_{2} \alpha\right\}\right\}$. In the case of large $\epsilon$, when $\epsilon>3 \sqrt{\alpha} \log n$, we have $\mathbb{P}\left\{\left|\overline{\mathcal{A}}_{l}\right|>C_{2} \alpha\right\} \leq 1 / 2 n^{4}$, for $C_{2} \geq \max \{e, 26 / \alpha\}$. In the case of small $\epsilon$, when $\epsilon \leq 3 \sqrt{\alpha} \log n$, we have $\mathbb{P}\left\{\left|\overline{\mathcal{A}}_{l}\right|>\max \left\{e^{-C_{1} \epsilon} m, C_{2} \alpha\right\}\right\} \leq 1 / 2 n^{4}$, for $C_{1} \leq 1 / 600 \sqrt{\alpha}$ and $C_{2} \geq 130$. Here we made a moderate assumption of $\epsilon \geq 3 \sqrt{\alpha}$, which is typically in the region of interest.

Analogously, we can prove that $\mathbb{P}\left\{\left|\overline{\mathcal{A}}_{r}\right|>\max \left\{e^{-C_{1} \epsilon} n, C_{2}\right\}\right\} \leq 1 / 2 n^{4}$, which finishes the proof of Remark 4.2,

\section{B Proof of Remark 4.4}

The expectation of the contribution of light couples, when each edge is independently revealed with probability $\epsilon / \sqrt{m n}$, is

$$
\mathbb{E}[Z]=\frac{\epsilon}{\sqrt{m n}}\left(\sum_{(i, j) \in L} x_{i} M_{i j}^{A} y_{j}-x^{T} M y\right)
$$

where we define $M^{A}$ by setting to zero the rows of $M$ whose index is not in $A_{l}$ and the columns of $M$ whose index is not in $A_{r}$.

In order to bound $\sum_{L} x_{i} M_{i j}^{A} y_{j}-x^{T} M y$, we write,

$$
\begin{aligned}
\left|\sum_{(i, j) \in L} x_{i} M_{i j}^{A} y_{j}-x^{T} M y\right| & =\left|x^{T}\left(M^{A}-M\right) y-\sum_{(i, j) \in \bar{L}} x_{i} M_{i j}^{A} y_{j}\right| \\
& \leq\left|x^{T}\left(M^{A}-M\right) y\right|+\left|\sum_{(i, j) \in \bar{L}} x_{i} M_{i j}^{A} y_{j}\right|
\end{aligned}
$$

Note that $\left|\left(M^{A}-M\right)_{i j}\right|$ is non-zero only if $i \notin A_{l}$ or $j \notin A_{r}$, in which case $\left|\left(M^{A}-M\right)_{i j}\right| \leq$ $M_{\text {max }}$. Also, by Remark 4.2, there exists $\delta=\max \left\{e^{-C_{1} \epsilon}, C_{2} / n\right\}$ such that $\left|i: i \notin A_{l}\right| \leq \delta m$ and $\left|j: j \notin A_{r}\right| \leq \delta n$. Denoting by $\mathbb{I}(\cdot)$ the indicator function, we have

$$
\begin{aligned}
\left|x^{T}\left(M^{A}-M\right) y\right| & \leq \sum_{i j}\left|x_{i}\right|\left|y_{j}\right|\left(\mathbb{I}\left(i \notin A_{l}\right)+\mathbb{I}\left(j \notin A_{r}\right)\right) M_{\max } \\
& =\left(\sum_{i}\left|x_{i}\right| \mathbb{I}\left(i \notin A_{l}\right) \sum_{j}\left|y_{j}\right|+\sum_{j}\left|y_{j}\right| \mathbb{I}\left(j \notin A_{r}\right) \sum_{i}\left|x_{i}\right|\right) M_{\max } \\
& \leq(\sqrt{\delta m} \sqrt{n}+\sqrt{\delta n} \sqrt{m}) M_{\max } \\
& \leq M_{\max } \sqrt{\frac{m n}{\epsilon}} .
\end{aligned}
$$


for $\delta \leq \frac{1}{4 \epsilon}$. We can bound the second term as follows

$$
\begin{aligned}
\left|\sum_{(i, j) \in \bar{L}} x_{i} M_{i j}^{A} y_{j}\right| & \leq \sum_{(i, j) \in \bar{L}} \frac{\left|x_{i} M_{i j}^{A} y_{j}\right|^{2}}{\left|x_{i} M_{i j}^{A} y_{j}\right|} \\
& \leq \frac{1}{M_{\max }} \sqrt{\frac{m n}{\epsilon}} \sum_{(i, j) \in \bar{L}}\left|x_{i} M_{i j}^{A} y_{j}\right|^{2} \\
& \leq \frac{1}{M_{\max }} \sqrt{\frac{m n}{\epsilon}} \sum_{i \in[m], j \in[n]}\left|x_{i} M_{i j}^{A} y_{j}\right|^{2} \\
& \leq M_{\max } \sqrt{\frac{m n}{\epsilon}},
\end{aligned}
$$

where the second inequality follows from the definition of heavy couples.

Hence, summing up the two contributions, we get

$$
|\mathbb{E}[Z]| \leq 2 M_{\max } \sqrt{\epsilon}
$$

\section{Proof of Remark 4.5}

We can associate to the matrix $Q$ a bipartite graph $\mathcal{G}=([m],[n], \mathcal{E})$. The proof is similar to the one in [FKS89, FO05] and is based on two properties of the graph $\mathcal{G}$ :

1. Bounded degree. The graph $\mathcal{G}$ has maximum degree bounded by a constant times the average degree:

$$
\begin{aligned}
\operatorname{deg}(i) & \leq \frac{2 \epsilon}{\sqrt{\alpha}}, \\
\operatorname{deg}(j) & \leq 2 \epsilon \sqrt{\alpha}
\end{aligned}
$$

for all $i \in[m]$ and $j \in[n]$.

2. Discrepancy. We say that $\mathcal{G}$ (equivalently, the adjacency matrix $Q$ ) has the discrepancy property if, for any $A \subseteq[m]$ and $B \subseteq[n]$, one of the following is true:

$$
\begin{aligned}
& \text { 1. } \frac{e(A, B)}{\mu(A, B)} \leq \xi_{1}, \\
& \text { 2. } e(A, B) \ln \left(\frac{e(A, B)}{\mu(A, B)}\right) \leq \xi_{2} \max \{|A| / \sqrt{\alpha},|B| \sqrt{\alpha}\} \ln \left(\frac{\sqrt{m n}}{\max \{|A| / \sqrt{\alpha},|B| \sqrt{\alpha}\}}\right) .
\end{aligned}
$$

for two numerical constants $\xi_{1}, \xi_{2}$ (independent of $n$ and $\epsilon$ ). Here $e(A, B)$ denotes the number of edges between $A$ and $B$ and $\mu(A, B)=|A||B||E| / m n$ denotes the average number of edges between $A$ and $B$ before trimming.

We will prove, later in this section, that the discrepancy property holds with high probability.

Let us partition row and column indices with respect to the value of $x_{u}$ and $y_{v}$ :

$$
\begin{aligned}
& A_{i}=\left\{u \in[m]: \frac{\Delta}{\sqrt{m}} 2^{i-1} \leq\left|x_{u}\right|<\frac{\Delta}{\sqrt{m}} 2^{i}\right\}, \\
& B_{j}=\left\{v \in[n]: \frac{\Delta}{\sqrt{n}} 2^{j-1} \leq\left|y_{v}\right|<\frac{\Delta}{\sqrt{n}} 2^{j}\right\},
\end{aligned}
$$


for $i \in\{1,2, \ldots,\lceil\ln (\sqrt{m} / \Delta) / \ln 2\rceil\}$, and $j \in\{1,2, \ldots,\lceil\ln (\sqrt{n} / \Delta) / \ln 2\rceil\}$, and we denote the size of subsets $A_{i}$ and $B_{j}$ by $a_{i}$ and $b_{j}$ respectively. Furthermore, we define $e_{i, j}$ to be the number of edges between two subsets $A_{i}$ and $B_{j}$, and we let $\mu_{i, j}=a_{i} b_{j}(\epsilon / \sqrt{m n})$. Notice that all indices $u$ of non zero $x_{u}$ fall into one of the subsets $A_{i}$ 's defined above, since, by discretization, the smallest non-zero element of $x \in T_{m}$ in absolute value is at least $\Delta / \sqrt{m}$. The same applies for the entries of $y \in T_{n}$.

By grouping the summation into $A_{i}$ 's and $B_{j}$ 's, we get

$$
\begin{aligned}
\sum_{\substack{(u, v): \\
\left|x_{u} y_{v}\right| \geq \frac{C \sqrt{\epsilon}}{\sqrt{m n}}}} Q_{u v}\left|x_{u} y_{v}\right| & \leq \sum_{(i, j): 2^{i+j} \geq \frac{4 C \sqrt{\epsilon}}{\Delta^{2}}} e_{i, j} \frac{\Delta 2^{i}}{\sqrt{m}} \frac{\Delta 2^{j}}{\sqrt{n}} \\
& =\Delta^{2} \sum a_{i} b_{j} \frac{\epsilon}{\sqrt{m n}} \frac{e_{i, j}}{\mu_{i, j}} \frac{2^{i}}{\sqrt{m}} \frac{2^{j}}{\sqrt{n}} \\
& =\Delta^{2} \sqrt{\epsilon} \sum \underbrace{a_{i} \frac{2^{2 i}}{m}}_{\alpha_{i}} \underbrace{b_{j} \frac{2^{2 j}}{n}}_{\beta_{j}} \underbrace{\frac{e_{i, j} \sqrt{\epsilon}}{\mu_{i, j} 2^{i+j}}}_{\sigma_{i, j}} .
\end{aligned}
$$

Note that, by definition, we have

$$
\begin{aligned}
& \sum_{i} \alpha_{i} \leq 4\|x\|^{2} / \Delta^{2}, \\
& \sum_{i} \beta_{i} \leq 4\|y\|^{2} / \Delta^{2} .
\end{aligned}
$$

We are now left with task of bounding $\sum \alpha_{i} \beta_{j} \sigma_{i, j}$, for $Q$ that satisfies bounded degree property and discrepancy property.

Define,

$$
\begin{aligned}
& \mathcal{C}_{1} \equiv\left\{(i, j): 2^{i+j} \geq \frac{4 C \sqrt{\epsilon}}{\Delta^{2}} \text { and }\left(A_{i}, B_{j}\right) \text { satisfies (71) }\right\} \\
& \mathcal{C}_{2} \equiv\left\{(i, j): 2^{i+j} \geq \frac{4 C \sqrt{\epsilon}}{\Delta^{2}} \text { and }\left(A_{i}, B_{j}\right) \text { satisfies (72) }\right\} \backslash \mathcal{C}_{1} .
\end{aligned}
$$

We need to show that $\sum_{(i, j) \in \mathcal{C}_{1} \cup \mathcal{C}_{2}} \alpha_{i} \beta_{j} \sigma_{i, j}$ is bounded.

For the terms in $\mathcal{C}_{1}$ this bound is easy. Since summation is over pairs of indices $(i, j)$ such that $2^{i+j} \geq \frac{4 C \sqrt{\epsilon}}{\Delta^{2}}$, it follows from bounded degree property that $\sigma_{i, j} \leq \xi_{1} \Delta^{2} / 4 C$. By Eqs. (73) and (74)), we have $\sum_{\mathcal{C}_{1}} \alpha_{i} \beta_{j} \sigma_{i, j} \leq\left(\xi_{1} \Delta^{2} / 4 C\right)(2 / \Delta)^{4}=O(1)$.

For the terms in $\mathcal{C}_{2}$ the bound is more complicated. We assume $a_{i} \leq \alpha b_{j}$ for simplicity and the other case can be treated in the same manner. By change of notation the second discrepancy condition becomes

$$
e_{i, j} \log \left(\frac{e_{i, j}}{\mu_{i, j}}\right) \leq \xi_{2} \max \left\{a_{i} / \sqrt{\alpha}, b_{j} \sqrt{\alpha}\right\} \log \left(\frac{\sqrt{m n}}{\max \left\{a_{i} / \sqrt{\alpha}, b_{j} \sqrt{\alpha}\right\}}\right) .
$$

We start by changing variables on both sides of Eq. (177).

$$
\frac{e_{i, j} a_{i} b_{j} \epsilon}{\mu_{i, j} \sqrt{m n}} \log \left(\frac{e_{i, j}}{\mu_{i, j}}\right) \leq \xi_{2} b_{j} \sqrt{\alpha} \log \left(\frac{2^{2 j}}{\beta_{j}}\right) .
$$


Now, multiply each side by $2^{i} / b_{j} \sqrt{\epsilon} 2^{j}$ to get

$$
\sigma_{i, j} \alpha_{i} \log \left(\frac{e_{i, j}}{\mu_{i, j}}\right) \leq \frac{\xi_{2} 2^{i}}{\sqrt{\epsilon} 2^{j}}\left[\log \left(2^{2 j}\right)-\log \beta_{j}\right] .
$$

To achieve the desired bound, we partition the analysis into 5 cases:

1. $\sigma_{i, j} \leq 1$ : By Eqs. (73) and (74), we have $\sum \alpha_{i} \beta_{j} \sigma_{i, j} \leq(2 / \Delta)^{4}=O(1)$.

2. $2^{i}>\sqrt{\epsilon} 2^{j}$ : By the bounded degree property in Eq. (70), we have $e_{i, j} \leq a_{i} 2 \epsilon / \sqrt{\alpha}$, which implies that $e_{i, j} / \mu_{i, j} \leq 2 n / b_{j}$. For a fixed $i$ we have, $\sum_{j} \beta_{j} \sigma_{i, j} \mathbb{I}\left(2^{i}>\sqrt{\epsilon} 2^{j}\right) \leq 2 \sqrt{\epsilon} \sum_{j} 2^{j-i} \mathbb{I}\left(2^{i}>\sqrt{\epsilon} 2^{j}\right) \leq$ 4. Then, $\sum \alpha_{i} \beta_{j} \sigma_{i, j} \leq 16 / \Delta^{2}=O(1)$.

3. $\log \left(e_{i, j} / \mu_{i, j}\right)>\frac{1}{4}\left[\log \left(2^{2 j}\right)-\log \beta_{j}\right]:$ From Eq.(178), it immediately follows that $\sigma_{i, j} \alpha_{i} \leq \frac{4 \xi_{2} 2^{i}}{\sqrt{\epsilon} 2^{j}}$. Due to case 2, we can assume $2^{i} \leq \sqrt{\epsilon} 2^{j}$, which implies that for a fixed $\mathrm{j}$ we have the following inequality : $\sum_{i} \sigma_{i, j} \alpha_{i} \leq 4 \xi_{2} \sum_{i} \frac{2^{i}}{\sqrt{\epsilon} 2^{j}} \mathbb{I}\left(2^{i} \leq \sqrt{\epsilon} 2^{j}\right) \leq 8 \xi_{2}$. Then it follows by Eq. (74) that $\sum \alpha_{i} \beta_{j} \sigma_{i, j} \leq 32 \xi_{2} / \Delta^{2}=O(1)$.

4. $\log \left(2^{2 j}\right) \geq-\log \beta_{j}$ : Due to case 3 , we can assume $\log \left(e_{i, j} / \mu_{i, j}\right) \leq \frac{1}{4}\left[\log \left(2^{2 j}\right)-\log \beta_{j}\right]$, which implies that $\log \left(e_{i, j} / \mu_{i, j}\right) \leq \log \left(2^{j}\right)$. Further, since we are not in case 1 , we can assume $1<\sigma_{i, j}=e_{i, j} \sqrt{\epsilon} / \mu_{i, j} 2^{i+j}$. Combining those two inequalities, we get $2^{i} \leq \sqrt{\epsilon}$.

Since in defining $\mathcal{C}_{2}$ we excluded $\mathcal{C}_{1}$, if $(i, j) \in \mathcal{C}_{2}$ then $\log \left(e_{i, j} / \mu_{i, j}\right) \geq 1$. Applying Eq. (78) we get $\sigma_{i, j} \alpha_{i} \leq \sigma_{i, j} \alpha_{i} \log \left(e_{i, j} / \mu_{i, j}\right) \leq\left(\xi_{2} 2^{i-j} / \sqrt{\epsilon}\right)\left[\log \left(2^{2 j}\right)-\log \beta_{j}\right] \leq 4 \xi_{2} 2^{i} / \sqrt{\epsilon}$.

Combining above two results, it follows that $\sum_{i} \sigma_{i, j} \alpha_{i} \leq 4 \xi_{2} \sum_{i} \frac{2^{i}}{\sqrt{\epsilon}} \mathbb{I}\left(2^{i} \leq \sqrt{\epsilon}\right) \leq 8 \xi_{2}$. Then, we have the desired bound : $\sum \alpha_{i} \beta_{j} \sigma_{i, j} \leq \frac{32 \xi_{2}}{\Delta^{2}}=O(1)$.

5. $\log \left(2^{2 j}\right)<-\log \beta_{j}$ : It follows, since we are not in case 3 , that $\log \left(e_{i, j} / \mu_{i, j}\right) \leq \frac{1}{4}\left[\log \left(2^{2 j}\right)-\log \beta_{j}\right] \leq$ $-\log \beta_{j}$. Hence, $e_{i, j} / \mu_{i, j} \leq 1 / \beta_{j}$. This implies that $\sigma_{i, j}=e_{i, j} \sqrt{\epsilon} / \mu_{i, j} 2^{i+j} \leq \sqrt{\epsilon} / \beta_{j} 2^{i+j}$. Since the summation is over pairs of indices $(i, j)$ such that $2^{i+j} \geq 4 C \sqrt{\epsilon} / \Delta^{2}$, we have $\sum_{j} \sigma_{i, j} \beta_{j} \leq \frac{\Delta^{2}}{2 C}$. Then it follows that $\sum \alpha_{i} \beta_{j} \sigma_{i, j} \leq \frac{2}{C}=O(1)$.

Analogous analysis for the set of indices $(i, j)$ such that $a_{i}>\alpha b_{j}$ will give us similar bounds. Summing up the results, we get that there exists a constant $C^{\prime} \leq \frac{32}{\Delta^{4}}+\frac{4 \xi_{1}}{C \Delta^{2}}+\frac{32}{\Delta^{2}}+\frac{128 \xi_{2}}{\Delta^{2}}+\frac{4}{C}$, such that

$$
\sum_{(i, j): 2^{i+j} \geq \frac{4 C \sqrt{\epsilon}}{\Delta^{2}}} \alpha_{i} \beta_{j} \sigma_{i, j} \leq C^{\prime} .
$$

This finishes the proof of Remark 4.5.

Lemma C.1. The adjacency matrix $Q$ has discrepancy property with probability at least $1-1 / 2 n^{3}$.

Proof. The proof is a generalization of analogous result in [FKS89, FO05] which is proved to hold only with probability larger than $1-e^{-C \epsilon}$. The stronger statement quoted here is a result of the observation that, when we trim the graph the number of edges between any two subsets does not increase. Define $Q_{0}$ to be the adjacency matrix corresponding to original random matrix $M^{E}$ before trimming. If the discrepancy assumption holds for $Q_{0}$, then it also holds for $Q$, since $e^{Q}(A, B) \leq e^{Q_{0}}(A, B)$, for $A \subseteq[m]$ and $B \subseteq[n]$.

Now we need to show that the desired property is satisfied for $Q_{0}$. This is proved for the case of non-bipartite graph in Section 2.2.5 of [FO05, and analogous analysis for bipartite graph shows 
that for all subsets $A \subseteq[m]$ and $B \subseteq[n]$, with probability at least $1-1 / 2(m n)^{p}$, the discrepancy condition holds with $\xi_{1}=2 e$ and $\xi_{2}=(3 p+12)\left(\alpha^{1 / 2}+\alpha^{-1 / 2}\right)$. Since we assume $\alpha \geq 1$, taking $p$ to be $3 / 2$ proves the desired thesis.

\section{Proof of remarks 6.1, 6.2 and 6.3}

Proof. (Remark 6.1.) Let $\theta=\left(\theta_{1}, \ldots, \theta_{p}\right), \theta_{i} \in[-\pi / 2, \pi / 2]$ be the principal angles between the planes spanned by the columns of $X_{1}$ and $X_{2}$. It is known that $d_{\mathrm{c}}\left(X_{1}, X_{2}\right)=\|2 \sin (\theta / 2)\|_{2}$ and $d_{\mathrm{p}}\left(X_{1}, X_{2}\right)=\|\sin \theta\|_{2}$. The thesis follows from the elementary inequalities

$$
\frac{1}{\pi} \alpha \leq \sqrt{2} \sin (\alpha / 2) \leq \sin \alpha \leq 2 \sin (\alpha / 2)
$$

valid for $\alpha \in[0, \pi / 2]$.

Proof. (Remark 6.2) Given $X \in \mathbb{R}^{n \times r}$, define $X^{\prime}$ by

$$
X^{\prime(i)}=\frac{X^{(i)}}{\left\|X^{(i)}\right\|} \min \left(\left\|X^{(i)}\right\|, \sqrt{\mu_{0} r}\right)
$$

for all $i \in[n]$.

Let $A$ be a matrix for extracting the ortho-normal basis of the columns of $X^{\prime}$. That is $A \in \mathbb{R}^{r \times r}$ such that $X^{\prime \prime}=X^{\prime} A$ and $X^{\prime \prime T} X^{\prime \prime}=n \mathbf{1}$. Without loss of generality, $A$ can be taken to be a symmetric matrix. In the following, let $\sigma_{i}=\sigma_{i}\left(A^{-1}\right)$ for all $i \in[n]$. Note that by construction $d\left(U, X^{\prime}\right) \leq d(U, X) \leq \delta$. Hence there is a $Q_{1} \in \mathrm{O}(r)$ such that,

$$
\left\|U-X^{\prime} Q_{1}\right\|_{F}^{2} \leq n \delta^{2}
$$

We start by writing

$n A^{-T} A^{-1}=X^{\prime T} X^{\prime}=Q_{1}\left(n \mathbf{1}-\left(U-X^{\prime} Q_{1}\right)^{T} U+U^{T}\left(U-X^{\prime} Q_{1}\right)+\left(U-X^{\prime} Q_{1}\right)^{T}\left(U-X^{\prime} Q_{1}\right)\right) Q_{1}^{T} .(81$

Using (80), we have

$$
\begin{aligned}
\left\|\left(U-X^{\prime} Q_{1}\right)^{T} U\right\|_{F} & \leq\|U\|_{2}\left\|\left(U-X^{\prime} Q_{1}\right)\right\|_{F} \\
& \leq n \delta
\end{aligned}
$$

and

$$
\left\|\left(U-X^{\prime} Q_{1}\right)^{T}\left(U-X^{\prime} Q_{1}\right)\right\|_{F} \leq n \delta^{2} .
$$

Therefore, using (81)

$$
\begin{aligned}
& \sigma_{1}^{2} \leq 1+2 \delta+\delta^{2} \\
& \sigma_{r}^{2} \geq 1-2 \delta-\delta^{2} .
\end{aligned}
$$

From (82), (83) and $\delta \leq 1 / 16$, we get $\sigma_{1} \leq \sqrt{3}$ and $\sigma_{r} \geq 1 / \sqrt{3}$. Since $\left\|X^{\prime \prime(i)}\right\|^{2}=\left\|X^{\prime(i)} A\right\|^{2} \leq 3 \mu_{0} r$ for all $i \in[n]$, we have that $X^{\prime \prime} \in \mathcal{K}\left(3 \mu_{0}\right)$. 
We next prove that $d\left(X^{\prime}, X^{\prime \prime}\right) \leq 3 \delta$ which implies the thesis by triangular inequality.

$$
\begin{aligned}
d\left(X^{\prime}, X^{\prime \prime}\right)^{2} & =\frac{1}{n} \min _{Q \in \mathrm{O}(r)}\left\|X^{\prime}-X^{\prime \prime} Q\right\|_{F}^{2} \\
& \leq \frac{1}{n}\left\|X^{\prime \prime} A^{-1}-X^{\prime \prime}\right\|_{F}^{2} \\
& =\left\|A^{-1}-\mathbf{1}\right\|_{F}^{2} \\
& \leq\left\|\left(A^{-1}-\mathbf{1}\right)\left(A^{-1}+\mathbf{1}\right)\right\|_{F}^{2} \\
& \leq\left\|A^{-T} A^{-1}-\mathbf{1}\right\|_{F}^{2} \\
& \leq 9 \delta^{2}
\end{aligned}
$$

where the last inequality is from (81).

Proof. (Remark 6.3.) We start by observing that

$$
d_{\mathrm{p}}(V, Y)=\frac{1}{\sqrt{n}} \min _{A \in \mathbb{R}^{r \times r}}\|V-Y A\|_{F} .
$$

Indeed the minimization on the right hand side can be performed explicitly (as $\|V-Y A\|_{F}^{2}$ is a quadratic function of $A$ ) and the minimum is achieved at $A=Y^{T} V / n$. The inequality follows by simple algebraic manipulations.

Take $A=S^{T} X^{T} U \Sigma^{-1} / m$. Then

$$
\begin{aligned}
\|V-Y A\|_{F} & =\sup _{B,\|B\|_{F} \leq 1}\langle B,(V-Y A)\rangle \\
& =\sup _{B,\|B\|_{F} \leq 1}\left\langle B^{T}, \frac{1}{m} \Sigma^{-1} U^{T}\left(U \Sigma V^{T}-X S Y^{T}\right)\right\rangle \\
& =\frac{1}{m} \sup _{B,\|B\|_{F} \leq 1}\left\langle U \Sigma^{-1} B^{T},(M-\widehat{M})\right\rangle \\
& \leq \frac{1}{m} \sup _{B,\|B\|_{F} \leq 1}\left\|U \Sigma^{-1} B^{T}\right\|_{F}\|M-\widehat{M}\|_{F} .
\end{aligned}
$$

On the other hand

$$
\left\|U \Sigma^{-1} B^{T}\right\|_{F}^{2}=\operatorname{Tr}\left(B \Sigma^{-1} U^{T} U \Sigma^{-1} B^{T}\right)=m \operatorname{Tr}\left(B^{T} B \Sigma^{-2}\right) \leq m \Sigma_{\min }^{-2}\|B\|_{F}^{2},
$$

whereby the last inequality follows from the fact that $\Sigma$ is diagonal. Together (84) and (88), this implies the thesis.

\section{References}

$\left[\mathrm{AFK}^{+} 01\right]$ Y. Azar, A. Fiat, A. Karlin, F. McSherry, and J. Saia, Spectral analysis of data, Proceedings of the thirty-third annual ACM symposium on Theory of computing (New York, NY, USA), ACM, 2001, pp. 619-626.

[AM07] D. Achlioptas and F. McSherry, Fast computation of low-rank matrix approximations, J. ACM 54 (2007), no. 2, 9.

[Arm66] L. Armijo, Minimization of functions having lipschitz continuous first partial derivatives, Pacific J. Math. 16 (1966), no. 1, 1-3. 
[BDJ99] M. W. Berry, Z. Drmać, and E. R. Jessup, Matrices, vector spaces, and information retrieval, SIAM Review 41 (1999), no. 2, 335-362.

[Ber92] M. W. Berry, Large scale sparse singular value computations, International Journal of Supercomputer Applications 6 (1992), 13-49.

[CCS08] J-F Cai, E. J. Candès, and Z. Shen, A singular value thresholding algorithm for matrix completion, arXiv:0810.3286, 2008.

[CR08] E. J. Candès and B. Recht, Exact matrix completion via convex optimization, arxiv:0805.4471, 2008.

[CRT06] E. J. Candes, J. K. Romberg, and T. Tao, Robust uncertainty principles: exact signal reconstruction from highly incomplete frequency information, IEEE Trans. on Inform. Theory 52 (2006), 489- 509.

[CT09] E. J. Candès and T. Tao, The power of convex relaxation: Near-optimal matrix completion, arXiv:0903.1476, 2009.

[Don06] D. L. Donoho, Compressed Sensing, IEEE Trans. on Inform. Theory 52 (2006), 12891306 .

[EAS99] A. Edelman, T. A. Arias, and S. T. Smith, The geometry of algorithms with orthogonality constraints, SIAM J. Matr. Anal. Appl. 20 (1999), 303-353.

[Faz02] M. Fazel, Matrix rank minimization with applications, Ph.D. thesis, Stanford University, 2002.

[FKS89] J. Friedman, J. Kahn, and E. Szemerédi, On the second eigenvalue in random regular graphs, Proceedings of the Twenty-First Annual ACM Symposium on Theory of Computing (Seattle, Washington, USA), ACM, may 1989, pp. 587-598.

[FKV04] A. Frieze, R. Kannan, and S. Vempala, Fast monte-carlo algorithms for finding low-rank approximations, J. ACM 51 (2004), no. 6, 1025-1041.

[FO05] U. Feige and E. Ofek, Spectral techniques applied to sparse random graphs, Random Struct. Algorithms 27 (2005), no. 2, 251-275.

[KMO08] R. H. Keshavan, A. Montanari, and S. Oh, Learning low rank matrices from $O(n)$ entries, Proc. of the Allerton Conf. on Commun., Control and Computing, September 2008.

[KOM09] R. H. Keshavan, S. Oh, and A. Montanari, Matrix completion from a few entries, arXiv:0901.3150, January 2009.

[Net] Netflix prize, http://www.netflixprize.com/.

[RFP07] B. Recht, M. Fazel, and P. Parrilo, Guaranteed minimum rank solutions of matrix equations via nuclear norm minimization, arxiv:0706.4138, 2007.

[SC09] A. Singer and M. Cucuringu, Uniqueness of low-rank matrix completion by rigidity theory, arXiv:0902.3846, January 2009. 